\title{
La crise exemplaire d'un modèle européen en Amérique latine: les racines anciennes du retrait du modèle politique et culturel français
}

Denis ROLLAND

Résumé: Français et francophiles ont abondamment glosé depuis plus d'un siècle sur le caractère déterminant de l'influence française dans la naissance des nouveaux Etats du souscontinent latino-américain. Aujourd'hui cependant, une antienne revient souvent dans ce Nouveau Monde: des Révolutions d'Indépendance et de la naissance des Etats-nations américains à nos jours, l'Amérique latine aurait progressivement changé de sphère culturelle, largué les amarres de l'Europe vieillissante ancrée, en particulier la France, dans un passé fastueux. $\mathrm{Ce}$ phénomène supposé requiert l'analyse.

Mots-clés: Francophiles; Influence française; Amerique Latine.

Français et francophiles ont abondamment glosé depuis plus d'un siècle sur le caractère déterminant de l'influence française dans la naissance des nouveaux Etats du souscontinent latino-américain. Aujourd'hui cependant, une antienne revient souvent dans ce Nouveau Monde: des Révolutions d'Indépendance et de la naissance des Etats-nations américains à nos jours, l'Amérique latine aurait progressivement changé de sphère culturelle, largué les amarres de l'Europe vieillissante ancrée, en particulier la France, dans un passé fastueux. Ce phénomène supposé requiert l'analyse.

Dès avant la fin de la première moitié du dix-neuvième siècle et jusqu'au début du siècle suivant, l'Europe et la France

\footnotetext{
* Inst. d'Etudes Politiques de Paris - Université Robert Schuman 67045 - Strasbourg - França.
} 
sont omniprésentes en Amérique latine dans le discours et l'ensemble de la sphère publique. En France, ce "messianisme " français, selon le mot d'Albert Salon, est puissant, conscient, réfléchi ${ }^{1}$. Et l'argumentation paraît imparable : presque tout le sous-continent latin, Brésil excepté, a suivi dans le passage à la modernité politique une voie de rupture plaçant les nouveaux Etats, en matière de référence, dans la mouvance de la Révolution française. Peu importe alors que la généalogie de certaines certitudes soit en ce domaine hasardeuse ${ }^{2}$, cette référence drue, implicite et construite, appartient au patrimoine de la vie politique et culturelle de la plupart des Etats à l'orée du vingtième siècle et la représentation est créatrice de réalité.

Etablie par les historiens à de multiples reprises, cette présence dans l'espace public (et privé dans une moindre mesure) latino-américain ouvre la perspective de ces pages qui interrogent le XXe siècle pour comprendre le reflux d'un modèle universaliste et d'une influence européenne importante.

Mais, parce que l'historien bute souvent sur de trop grandes certitudes quant à l'influence passée de la France, parce qu'une part des liens (et des illusions mutuellement entretenues sur ces liens) entre France et Amérique latine semble se dissiper dès les années 1920 et plus nettement avec la Seconde Guerre mondiale, parce que la réciproque et persistante rhétorique d'amitié, dont les chaleureuses déclarations "mexicaines" des Présidents de Gaulle en 1964 ou Mitterrand en 1981, cache de plus en plus mal la toile de fond effilochée des relations entre France et Amérique latine, c'est dans la première moitié du siècle qu'il faut chercher les mécanismes de la crise du modèle français au vingtième siècle, avec quelques éléments de sa production, de sa circulation et de sa réception.

\section{LE CORPUS DES IDÉES FRANÇAISES : DES APPARENCES CONSTRUCTRICES DE RÉALITÉ AU DÉBUT DU XX $\mathrm{X}^{\mathrm{E}} \mathrm{S}$.}

Le mot "modèle" a de nombreuses limites qu'il ne convient pas d'analyser ici $^{3}$. Par contre, vue d'Amérique latine, 
dans le contexte plus général du rapport des élites aux modèles européens ${ }^{4}$, la France est considérée comme participant au premier chef de cette matrice supposée, fonctionnant souvent comme paradigme. S'identifier à la France ou à l'Europe par les idées, la pratique politique, culturelle ou sociale, le comportement ou le vêtement, conduit une large part des élites latino-américaines à une forte mimétique des apparences européennes au début du XXe siècle. Comme l'écrit en 1954, non sans caractère réducteur, Lucien Febvre: "Reportons-nous à cinquante ou soixante ans en arrière : l'homme très intelligent, très cultivé, en Amérique, à quoi aspirait-il ? Non pas à être Brésilien, mais à être, au Brésil, le représentant de la plus haute et de la plus fine culture intellectuelle" ${ }^{5}$, comprenons européenne et en particulier française.

Selon une chronologie complexe et variable d'un pays à un autre, l'image dominante ${ }^{6}$ de la France en Amérique latine comme l'afrancesamiento hispano-américain ou le francesismo brésilien procèdent de la diffusion des idées des Lumières et des principes mis en place par la Révolution française ${ }^{7}$. En particulier parce que la Révolution française a rompu de façon totale et soudaine avec l'absolutisme, la monarchie et tout l'Ancien Régime hiérarchique; parce qu'elle a construit le modèle théorique et abstrait d'une république idéale fondée sur la modernité à l'état pur; parce qu'en Amérique espagnole ${ }^{8}$, avec les indépendances et l'adoption de la forme républicaine qui place les nouveaux Etats dans le droit fil de la Révolution française et dans la logique libérale, on constate le triomphe d'une modernité théorique plus pure même qu'en France, revenue au cadre de la monarchie constitutionnelle après l'Empire ${ }^{9}$. En outre, en Amérique latine, contrairement à ce qui se passe en Europe, l'appartenance à la nation ne peut être définie par le sang ou, sauf au Brésil, par la langue : au moment des indépendances, la nation ne peut être qu'un projet, organisé, pensé par les élites, prenant référence notamment au Contrat social de Jean-Jacques Rousseau et s'appuyant sur l'idée d'une nation formée d'individus libres et égaux décidant de former 
cette nation. Le modèle du nationalisme latino-américain est ainsi, de fait, fortement lié aux Lumières.

\section{L'intrumentalisation des concordances chronologiques}

Entre Amérique latine et France, la concordance des moments culturels et politiques paraît justifier l'influence supposée déterminante de la France dans la naissance et le développement des Etats et des nations latino-américains. C'est l'un des discours les communs tenu par une large partie des historiens et des élites latino-américaines de la fin du XIXe siècle pour expliquer l'Indépendance du sous-continent. Et ce discours est constructeur de réalité puisqu'au début du siècle suivant et parfois bien plus tard, peu nombreux sont ceux, des deux côtés de l'Atlantique, qui s'interrogent sur la réalité de l'influence française dans l'histoire des Etats-nations latinoaméricains. Un Latino-Américain déclare dans un amphithéâtre de la Sorbonne avant la Première Guerre mondiale: "Notre histoire se développe parallèlement à la vôtre. Nous avons aussi nos Girondins, votre Déclaration des Droits est traduite à l'heure tragique de notre Indépendance. Bolivar médite le Contrat Social dans les premières années de sa mission libératrice. Bilbao demande des inspirations à Edgar Quinet, Montalvo exalte la démocratie chrétienne de Lamartine. Quand la dissolution nous menace, Guizot sera le maître de nos conservateurs angoissés. Auguste Comte donne une religion aux hommes d'Etat du Brésil qui avaient abandonné leurs vieux dogmes " ${ }^{10}$. Après la Seconde Guerre mondiale, le politologue français Charles Morazé écrit sans hésitation qu' "au Brésil les vicissitudes politiques sont exactement les nôtres, à l'échelle près, à leur position près dans le temps et dans l'espace "11.

Schématiquement, on peut, avec François-Xavier Guerra, distinguer quatre phases ${ }^{12}$.

Une première phase, lors des indépendances hispanoaméricaines, correspond assez bien à la Révolution française qui 
peut alors être construite comme initiatrice fondamentale. De 1808 à 1810, "on passe d'un régime absolutiste [...] à la proclamation par tous les pays hispaniques de la souveraineté nationale comme principe de légitimité ". Et, vers 1812-1813, les références politiques modernes s'imposent dans le monde hispanique, avec "partout, des Constitutions écrites, entièrement nouvelles, de coupe française "13. Le cas du Brésil est évidemment singulier: même si le "pouvoir royal" de Benjamin Constant inspire la conception d'un empereur "modérateur", lorsque Dom Pedro Ier est proclamé en 1822 empereur constitutionnel d'un Brésil uni, le modèle, s'il y a parce que l'Etat portugais n'a jamais cessé de fonctionner, est bien plus anglais que français.

Puis vient une phase doctrinaire en Amérique, laquelle concorde avec la Monarchie de Juillet, avec une restriction du suffrage. Importée de France et d'Espagne, "dans le Chili, l'Argentine, et le Mexique des années 1830, la théorie de la souveraineté rationnelle, la distinction entre souveraineté du peuple et souveraineté nationale et le suffrage capacitaire" semblent fournir un modèle largement utilisé par les élites hispano-américaines ${ }^{14}$. Au Brésil, l'indépendance est complète lorsqu'en 1831 dom Pedro abdique en faveur de son fils mineur né au Brésil ; la concordance des chronologies entre Brésil et France, deux monarchies constitutionnelles, est alors un peu plus visible avec une monarchie bragantine liée aux Orléans ${ }^{15}$. Mais si certaines institutions sont inspirées de modèles français, comme la Garde nationale, le libéralisme qui entre en scène au Brésil est à nouveau plus influencé par le domaine anglo-saxon.

Une vague d'aspiration au suffrage universel et à la démocratisation des institutions déferle ensuite: elle touche, avec les révolutions de 1848, aussi bien la France que l'Allemagne, l'Autriche ou l'Amérique latine. A quelques exceptions près (Bolivie, Equateur, Pérou en raison de la forte présence indienne, et Colombie qui supprime le suffrage universel en 1886 après l'avoir adopté en 1853), le suffrage universel se généralise en terre hispano-américaine: en Argentine en 1853, au Mexique en 1856-1857, au Venezuela en 
1858, au Chili le processus engagé en 1849 aboutit en 1887. Point d'évolution comparable cependant au Brésil qui conserve des élections indirectes et un suffrage restreint.

La dernière phase, majeure sans doute, correspond au positivisme. Ce terme recouvre en Amérique latine des idées diverses, parfois fort différentes de celles d'Auguste Comte ${ }^{16}$. Mais, après les vagues de l'utilitarisme et du libéralisme, le positivisme parvient à dominer dans beaucoup de pays ${ }^{17}$ la pensée de l'élite latino-américaine à la fin du dix-neuvième siècle ${ }^{18}$. Il est très lié au modèle proposé par la France. Mais, dès l'origine, le comtisme politique est extrêmement ambigu par rapport aux valeurs qui sous-tendent le modèle français dominant: pour Comte, la Révolution a ainsi détruit les fondements du lien social et la société est menacée de désordre. En Amérique latine néanmoins, les positivistes républicains trouvent dans la pensée d'Auguste Comte un idéal de "scientificité ", bien qu'agissant " de manière pragmatique sans vouloir à tout prix restaurer l'unité perdue de la société "19. Au Mexique, Gabino Barreda, ministre de l'Education tente de réorganiser l'enseignement supérieur à partir des propositions sur la hiérarchie des sciences de Comte. Au Venezuela, à l'époque de l'autoritaire et cultivé Président Guzmán Blanco (1870-1888), la jeune élite accueille les nouvelles idées venues d'Europe ${ }^{20}$. Au Brésil, "la poussée des intellectuels s'effectue, dans une large mesure, sous le signe de la science "21 : là, le positivisme nourrit un fort courant de contestation politique et fournit les instruments conceptuels à l'instauration de la République. En 1889, la devise Ordem e Progresso est inscrite sur le drapeau national du Brésil républicain et un "positivisme intégral " est mis en œuvre.

L'historien cerne là le caractère spontané, non étatique, de cette diffusion, et donc du modèle : car toute la correspondance atteste que les diplomates de la République française apprécient peu cette image "subversive" de la France au Brésil et que l'adaptation du gouvernement républicain français à la nouvelle république est paradoxalement lente. A l'inverse, on distingue partout en Amérique latine des représentations différentes de la 
France, jacobine d'une part, positiviste de l'autre, auxquelles il faut ajouter celle, générale, aussi idéale qu'indécise d'une France rêvée qui participe à la formation de l'imaginaire populaire républicain, fortement au Brésil. Appuyé souvent sur un libéralisme politique ${ }^{22}$ dont la France n'a pas le monopole d'inspiration (au Brésil, il est largement d'origine nordaméricaine), le positivisme irrigue les élites latino-américaines de la fin du dix-neuvième siècle (et de plusieurs décennies encore par l'enseignement). A tel point qu'il constitue, ultérieurement parfois, comme au Mexique, l'un des arguments fédérateurs des oppositions aux régimes en place jugés antinationaux, afrancesados et cosmopolites ${ }^{23}$.

Ces conjonctures politiques et culturelles apparemment communes ont été maintes fois constatées entre France et Amérique latine depuis l'indépendance. Elles ont ensuite été instrumentalisées et répétées, à tel point que l'historien peut établir une véritable généalogie de ce type de discours. Or l'influence française est peut-être d'abord un discours sur un passé reconstitué. Elle participe du processus d'invention d'une tradition afin de satisfaire aux besoins du présent, puissamment renforcée par une instrumentalisation tardive. Le procédé n'a pas tout à fait cessé d'être répété. Ainsi, en introduction à l'une des manifestations du Bicentenaire de la Révolution française consacrée aux révolutions dans le monde ibérique, un historien nord-américain écrit en première phrase d'un article " en 1789, le monde entier avait les yeux fixés sur Paris"; il poursuit, à propos des premiers Etats-nations créés hors de l'Europe occidentale après 1789, en affirmant que “ c'est à la Révolution qu'ils devaient, en partie, leur naissance et l'essentiel des idéaux qu'ils professaient"; puis il conclut: "Les pays ibériques et l'Amérique latine rejetèrent les actes, mais flirtèrent avec les idées ". Mais il termine en citant un historien uruguayen de la fin du dix-neuvième siècle ${ }^{24}$, insistant sur " la contribution de la France à la culture universelle "25.

Certes, l'influence directe de la France a, durant ces dernières décennies, été sensiblement revue à la baisse. 
Le rôle postérieur des historiens latino-américains a été souligné, en particulier ceux de la seconde moitié du dixneuvième siècle. Dans la construction des histoires nationales, l'influence des historiens français a accentué le rôle supposé des idées venues de France : ils fournirent une grille d'interprétation historique jugée comme étant la seule à pouvoir fonder la légitimité des régimes politiques libéraux.

L'on sait aussi en Europe, au moins depuis la publication (1816-1831) du Voyage aux régions équinoxiales du Nouveau Continent d'Alexandre de Humboldt, que l'insurrection des populations noires de la colonie française de Saint-Domingue en août 1791 a beaucoup inquiété des élites créoles américaines nullement soucieuses de révolution sociale. Au Brésil, à Recife en 1824, noirs et mulâtres manifestent en chantant dans les rues, suscitant une peur profonde des élites: "Comme moi j'imite Christophe / Cet immortel Haïtien / Allons imite son peuple / Ô, mon peuple souverain ! " ${ }^{26}$.

De même, l'expérience de la Terreur, la "dérive jacobine " de la Révolution et, dans certains cas, le "despotisme" napoléonien conduisirent nombre de Latino-Américains à penser que les principes politiques de la révolution française avaient été mal appliqués et, parfois (comme Miranda), à rechercher, avec d'autres références (anglaises notamment), une forme de gouvernement " où le respect des lois rende compatibles l'ordre et la liberté "27.

Des travaux récents ont, de surcroît, souligné en Amérique andine, tant "la greffe absolutiste" à partir de 1770, que la perception d'une Révolution française satanique ${ }^{28}$. Et les polémiques françaises ont traversé l'Atlantique. Les articles de Ferdinand Brunetière critiquant violemment le positivisme traversent l'Océan en quelques semaines. Tous ces constats ont contribué à décaper le modèle hérité d'une historiographie libérale latino-américaine aux liens étroits, à la fin du dixneuvième siècle, avec la production scientifique de la Troisième République. Etablies récemment, ces modulations de l'analyse des racines de l'influence au moment des indépendances américaines, ne sont pas déterminantes: l'image construite 
durant le dix-neuvième siècle, en partie élaborée et diffusée par ces historiens latino-américains et durablement présente des deux côtés de l'Atlantique. Renan l'écrivait: “ L'oubli, et je dirai même l'erreur historique, sont un facteur essentiel de la création d'une nation "29.

Quoi qu'il en soit des origines réelles ou imaginées de cette influence de la France, au début du XXe siècle la France constitue un modèle communément revendiqué en Amérique latine par les élites, un modèle qui sert à se distinguer du reste de la société, politiquement et/ou socialement.

C'est aussi un modèle qui fonctionne par défaut. Les deux anciennes métropoles coloniales, Espagne et Portugal, ne peuvent être citées en référence dans les pays indépendants. La Grande-Bretagne, premier partenaire économique au XIXe siècle, est une monarchie: si elle peut servir de modèle pour certaines de ses institutions ou pour son fonctionnement politique, pour toutes les républiques hispano-américaines le modèle ne peut venir que d'une république, ou d'un pays qui a montré le chemin sur cette voie. L'Allemagne et l'Italie se constituent en tant qu'Etat tardivement et, longtemps. Les Etats-Unis, première république du continent, ont bien souvent servi de modèle institutionnel ou constitutionnel; mais leur politique continentale expansionniste manifeste à partir de 1848 (aux dépens du Mexique) font que la référence explicite et globale est dangereuse. Ainsi, la référence globale à la France, pays à la présence économique secondaire sur le continent, est fonctionnelle parce qu'elle est culturelle avant d'être politique, universaliste mais lointaine et donc peu dangereuse, et parce que le militantisme de la IIIe République fournit des modèles jugés partiellement transposables par les élites latinoaméricaines. 


\section{LOGIOUES ET MÉCANISMES D'UN ÉLOIGNEMENT}

\section{Une logique interne à l'Amérique latine}

Il y a tout d'abord une logique interne à l'Amérique latine, en deux termes au début du XXe siècle: la référence a un modèle extérieur n'est plus nécessaire ; et les valeurs qui purent être considérées comme "françaises " apparaissent de plus en plus naturalisées.

La France idéale renvoya longtemps à l'idée que l'Amérique latine se faisait d'elle-même : c'est ce qui fit la force de ce modèle français. Une grande partie des élites latinoaméricaines s'était donné au dix-neuvième siècle une double origine mythique: d'une part l'âge d'or des peuples d'avant la conquête; et, d'autre part, la société idéale proposée par la Révolution française. Néanmoins, les Etats construits et assurés, les logiques politiques latino-américaines requièrent peu à peu moins l'utilisation explicite de références culturelles extérieures, communes qui plus est. Les stratégies identitaires empruntent d'autres voies. A partir des années 1920 et 1930, le constat est souvent fait que la double origine mythique rend " plus difficile la solution des problèmes enracinés dans leurs réalités profondes ", et qu'il faut aussi prendre en compte l'histoire coloniale. La référence à la France perd une partie de son lien à la construction identitaire. Les rapports de la France et de l'Amérique latine peuvent évoluer progressivement vers un ordre plus conventionnel, celui de l'influence.

En outre, et quoi qu'il en soit du degré de fiction démocratique des fonctionnements politiques nationaux, la plupart des Etats tend à prôner officiellement l'attachement théorique aux principes issus des Lumières et de 1789. Mais, désormais, ils tendent à être considéré comme des valeurs nationales. Cela se constate même au Brésil, où, en raison de l'institution monarchique, l'Angleterre paraît avoir servi de modèle. Là, avant la proclamation tardive de la république en $1889 \mathrm{au}$ son de la Marseillaise, l'influence française est très forte, 
y compris dans les institutions politiques; et, après, elle ne signifie nullement soumission. Cette naturalisation des valeurs considérées comme d'origine françaises est claire même dans les plus jeunes Etats du continent, comme à Cuba où une fragile indépendance n'a été acquise qu'en 1902. Mettre un bonnet phrygien à l'emblème national est plus un signe d'appartenance à une modernité universelle qu'une référence précise à la France.

\section{Les signes du déclin autour de la Première Guerre mondiale}

Il y a d'abord la guerre comme échec.

La Première Guerre mondiale atteint marginalement l'Amérique latine. Et le positionnement des élites cultivées par rapport au conflit n'est pas manichéen, surtout au début. Mais au delà de cela, pour la France, être en guerre, c'est déjà un échec, même si les manifestations individuelles de soutien à la France et à l'Entente sont nombreuses et si, dans tous les pays, la guerre engendre un recrudescence de l'expression francophile. Certes, la propagande de l'Union sacrée française, avec son réinvestissement catholique explicite (Claudel est nommé ambassadeur pour cela à Rio pendant la guerre) conduit deux messianismes associés au sein de l'Union sacrée, le messianisme républicain et le messianisme catholique, à définir une dimension eschatologique de la culture de la France en guerre qui favorise une perception univoque à l'étranger. Au delà, l'Amérique latine voit la France en tête des vainqueurs ; et l'hypertrophie conjoncturelle d'institutions, tel le Comité FranceAmérique $^{30}$, ou la mise en place de missions militaires françaises dans plusieurs pays ${ }^{31}$ maintiennent des apparences brillantes. Ainsi, l'écho de la victoire alliée fournit des signes encourageants dans l'immédiat mais trompeurs et volatiles à moins court terme. Alors, comme une partie importante des opinions, la diplomatie, souvent affectée d'inertie culturelle, 
peine à prendre en compte le déclin, parce que, la rupture est surtout dans le regard de l'historien.

Mais la guerre, avant les incertitudes de l'Entre-deuxguerres en Europe, porte atteinte en Amérique latine aux représentations, en même temps qu'à la puissance économique, de la France, comme du reste de l'Europe, en Amérique latine. La guerre cristallise des évolutions, accélère des tendances sourdes. Derrière les défilés glorieux, se profile l'ébranlement de la perception de l'Europe : de même que la guerre marque une première étape du phénomène d'américanisation en France (cinéma, musique), l'extension, d'une dimension surtout continentale à une dimension mondiale, de la puissance militaire, commerciale et financière des Etats-Unis donne à ce concurrent un poids fondamental. Les Etats-Unis délèguent après la guerre, auprès du Brésil par exemple, des missions navales puis des missions aéronautiques : la "modernité " est ici, comme presque partout en Amérique latine, nord-américaine -dans le domaine militaire au moins. L'Europe, qui dictait le droit, apparaît inapte à gérer ses conflits autrement que par des voies militaires. Le monopole de l'Europe est brisé, surtout que les Nord-Américains ont largement contribué à l'issue du conflit. Le modèle républicain français semble mis en cause par la guerre.

Après " la guerre de 1914-1918, l'ascendant de la France a beaucoup diminué "32 en Amérique latine. Les critiques se multiplient, notamment contre le sentiment français de supériorité culturelle et son corollaire, le mépris pour le LatinoAméricain.

Avant la guerre, la présence française en Amérique latine était peu dynamique ou fragile. Après, le déclin de cette présence française est avéré ou renforcé.

La présence démographique française en Amérique latine était déjà très peu dynamique avant la Première Guerre : très peu de candidats français à l'émigration, intégration assez rapide dans la population du pays d'accueil... Tout cela manifestait au moins que le modèle français ne prenait pas appui sur une émigration notable, très faible relativement à 
d'autres communautés européennes, très inégalement répartie de surcroît.

\section{L'immigration occidentale au Brésil 1810-1915}

\begin{tabular}{|c|c|c|c|}
\hline & Nombre & total & Rang \\
\hline Italiens & 1361266 & 39,5 & 1 \\
\hline Portugais & 976386 & 28,3 & 2 \\
\hline Espagnols & 468583 & 13,6 & 3 \\
\hline Allemands & 122830 & 3,5 & 4 \\
\hline Russes & 103683 & 3,0 & 5 \\
\hline Autrichiens & 78546 & 2,3 & 6 \\
\hline Ottomans & 52435 & 1,5 & 7 \\
\hline Français & $\mathbf{2 8 0 7 2}$ & $\mathbf{0 , 8}$ & $\mathbf{8}$ \\
\hline Anglais & 22005 & 0,6 & 9 \\
\hline Total & 3213806 & 93,1 & \\
\hline Occidentaux & & & \\
\cline { 1 - 3 } & & &
\end{tabular}

Sources: D'après Revue de l'Amérique latine, 01-1923 et Gilles Matthieu, ouvr. cité, p. 22.

\section{La population française en Amérique latine (1912)}

\begin{tabular}{|c|c|c|c|c|}
\hline $\begin{array}{l}\text { Total général } \\
\text { Am. latine }\end{array}$ & \multicolumn{4}{|c|}{ 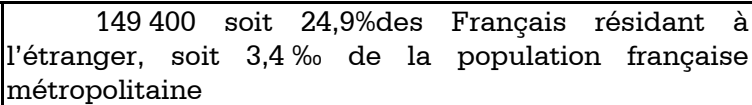 } \\
\hline Am. du Sud & 138000 & \multirow[t]{6}{*}{ dont } & Argentine & $\begin{array}{r}100000 \\
(67 \%) \\
\end{array}$ \\
\hline Caraïbes & 6300 & & Brésil & $\begin{array}{r}14000 \\
(9 \%)\end{array}$ \\
\hline $\begin{array}{l}\text { Am.centrale \& } \\
\text { Mexique }\end{array}$ & 5100 & & Chili & $\begin{array}{r}10000 \\
(7 \%) \\
\end{array}$ \\
\hline \multicolumn{2}{|c|}{ A titre de comparaison } & & Uruguay & $\begin{array}{r}9500 \\
(6 \%) \\
\end{array}$ \\
\hline Unis $^{\text {Etats- }}$ & 125000 & & Mexique & $(3 \%)<4000$ \\
\hline Canada & 25000 & & Cuba & 2300 \\
\hline
\end{tabular}

Sources : d'après Bulletin de la Statistique générale de la France, 011915, t.IV, fasc.2, p.163. 
Mais le phénomène est aggravé. Lors du conflit, contrairement à l'Allemagne qui a mobilisé ses ressortissants sur place ${ }^{33}$, préservant l'avenir et s'en servant pour sa propagande locale, la France, plus faible démographiquement, a voulu mobiliser tous les Français et cherché à les faire servir sur les fronts européens. Il y eut alors ceux qui se sont faits porter déserteurs, nombreux dans certains pays, franchissant une étape symbolique déterminante dans le processus d'assimilation à la nation d'accueil ; il y eut ceux qui moururent sur les champs de bataille : au Mexique, près du quart (21\%) des mobilisés a péri $^{34}$, des hommes en âge de travailler, non remplacés. D'autre part, la guerre accélère l'intégration de ces Français émigrés ; en témoigne le faible résultat de la mobilisation de 1939: au Mexique, moins de $15 \%$ de l'effectif mobilisable ${ }^{35}$; au Paraguay, $16 \%$, et à Cuba, il y a nettement plus d'insoumis que de mobilisés ${ }^{36}$. Enfin, les approvisionnements interrompus entre Amérique latine et France trouvent - pour la mode et les parfums, par exemple - des substitutions en provenance des Etats-Unis, même en ce qui concerne les commerces français installés en Amérique latine. Les flux commerciaux se réorientent vers le Nord et le public latino-américain prend durablement contact avec la production nord-américaine, d'autant qu'après manquent certains commis-voyageurs français.

Ainsi, la guerre affecte plus la présence économique française qu'une présence humaine déclinante. L'assise économique de la présence française était, avant 1914, déjà relativement fragile. Sans la modifier, elle renforce sa très inégale répartition géographique: en 1902, $59 \%$ de l'investissement français était concentré dans trois pays, Brésil, Argentine, Mexique ; en 1913, $89 \%$. Cette tendance persiste au delà. La guerre affecte encore plus le volume des échanges. Paul Claudel, nommé pendant la guerre à Rio, avait pour objectif de "placer le Brésil dans notre alliance et notre dépendance financière " 37 . Néanmoins, le désinvestissement français est net là comme ailleurs sur le continent. La dévaluation du franc français permit aux Latino-Américains de réduire leur dette. De 
plus, la guerre a coupé le souffle de l'investissement français en terre lointaine, souvent lié à l'agro-exportation. Surtout, la France, principal réservoir mondial de capitaux avant la guerre, a perdu définitivement, pour les besoins de la guerre, cette caractéristique au profit des Etats-Unis, devenus première puissance financière mondiale. Ce sont alors autant de points de contact entre élites économiques et politiques latinoaméricaines et françaises qui disparaissent. L'effort de guerre et les nécessités de la reconstruction dans les années 1920 ont, avant la crise de 1929, réduit la puissance financière de la France.

Placements et investissements français en Amérique latine 1902-1943 (millions de francs constants 1914. Classement 1913).

\begin{tabular}{|l|l|l|l|l|}
\hline Amérique du Sud & 2701 & 6182 & 639,2 & $-89,7$ \\
\hline Caraïbes & 210 & 120,5 & 11 & $-90,9$ \\
\hline Am.centale, Mexique & 341 & 2072,5 & 114,1 & $-94,5$ \\
\hline Total Amérique latine & 3252 & 8375 & 764,3 & $-90,9$ \\
\hline
\end{tabular}

Sources: d'après les données de "L'Amérique latine et la France", Notes et études documentaires, $\mathrm{n}^{\circ} 3084$, Paris, La Documentation française, 1964.

Entre 1919 et 1929, 35 \% des capitaux investis à l'étranger par les Etats-Unis se dirigent vers l'Amérique latine, provoquant une croissance de $125 \%$ du capital nord-américain dans le souscontinent. Même en comparant simplement avec l'Europe, la situation n'est pas toujours aussi positive qu'il y paraît : si, par exemple, Latécoère et Bouilloux-Laffont obtiennent en Uruguay, en Argentine et au Chili des concessions exclusives de lignes aériennes, même si au Brésil l'Aéropostale inaugure dès 1927 un vol régulier depuis la France, les Allemands (avec Dornier et Junkers) obtiennent là de meilleurs résultats. 
Investissements privés en Amérique latine (millions de dollars)

\begin{tabular}{|c|l|l|}
\hline Pays & 1897 & 1930 \\
\hline $\begin{array}{c}\text { Grande- } \\
\text { Bretagne }\end{array}$ & 2060 & 4500 \\
\hline France & 628 & 454 \\
\hline Allemagne & - & 700 \\
\hline Etats-Unis & 308 & 5429 \\
\hline
\end{tabular}

Source : Norman Bailey, Latin America in World Politics, 1967, p. 50 et Demetrio Boersner, Relaciones internacionales de América latina, Caracas, Nueva Sociedad, 5a ed, 1976, p. 138.

La crise commencée aux Etats-Unis en 1929 atteint de plein fouet les investissements français sur le continent. Les conséquences, comme pour les investissements britanniques, sont extrêmement importantes. Ainsi, en 1931, "l'empire" industriel Bouilloux-Lafont au Brésil, un des fleurons de l'investissement français, s'écroule ${ }^{38}$ tandis que la Banque hypothécaire franco-argentine vascille. Vis-à-vis de certains pays mono-exportateurs comme le Guatemala, le retrait de la France est proportionnellement plus fort que celui d'autres pays, dont l'Allemagne. Les Etats-Unis du New Deal, plus vite sortis de la crise que l'Europe et surtout la France, peuvent occuper les parcelles d'un marché de nouveau délaissé. En outre, lorsque le ciel de l'économie française se dégage un peu, la montée des périls en Europe ne permet pas d'inverser sensiblement la tendance.

Troisième signe de déclin autour de la Première Guerre mondiale, le recul de la destination française. Avant la guerre, les voyages en France des Latino-Américains étaient encore en progression. Ils sont, pour l'essentiel, suspendus pendant la guerre. Alors ces Latino-Américains privés d'Europe accentuent certains signes d'appropriation des héritages européens : la ville balnéaire de Mar del Plata en Argentine acquiert alors un aspect 
"européen" très marqué39. Lorsqu'ils reprennent après la Première Guerre mondiale, les voyages des Latino-Américains en France ne sont plus le fait, principalement, des élites économiques ou politiques - cas fréquent à la fin du dixneuvième siècle. Ce sont surtout les membres des élites littéraires ou artistiques qui traversent l'Atlantique parmi lesquels on retrouve Vicente Huidobro, Ricardo Güiraldes, Victoria Ocampo César Vallejo, Picabia, Carpentier... Cette évolution se conjugue avec un autre processus : la différentiation progressive entre élites culturelles, politiques et économiques. Un exemple de recul de cette destination française est donné par la formation des enfants du Président brésilien Getúlio Vargas : l'aîné des fils étudie en Allemagne, son second fait aux EtatsUnis des études d'ingénieur chimiste et sa fille, après une école anglaise, fait son droit au Brésil. L'Allemagne attire surtout les apprentis médecins, chimistes et ingénieurs ; l'Italie, juristes et artistes ; et ces deux Etats effectuent une promotion active en Amérique latine de leurs formations.

Certes, le graphique montre suivant que le nombre des Latino-Américains en France antérieur à la Guerre est de nouveau atteint au début des années 1930. Mais cette récupération dissimule plusieurs phénomènes. D'une part, elle est éphémère. D'autre part, le chiffre de 1931 doit être jugé à l'aune de la croissance démographique latino-américaine (60 millions d'habitants en 1900, 82 en 1914 et 111 en 1930), de l'élargissement de l'accès à la culture écrite et des facilités accrues de déplacement entre Amérique et Europe. 


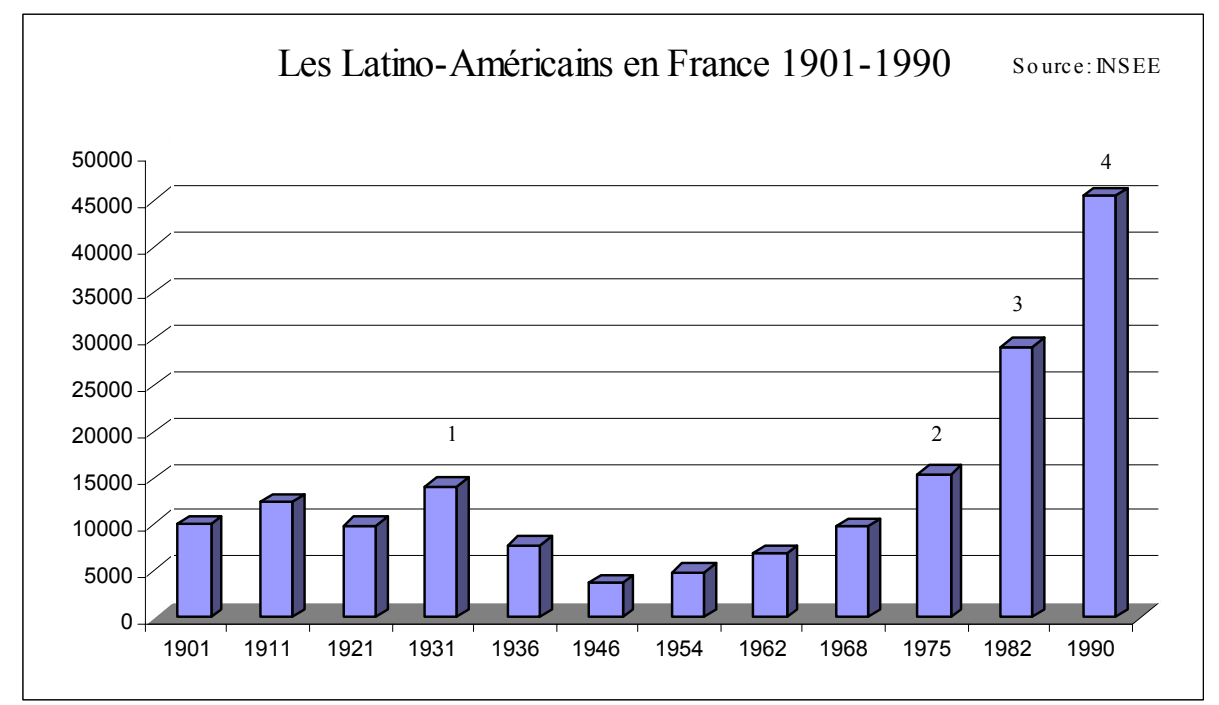

1. Dont 4047 Argentins et 1064 Brésiliens. 2. Depuis 1946, l'irrégularité des intervalles entre les recensements tend à minorer l'expression de la progression. 3. Dont 6272 Chiliens, 3808 Brésiliens, 4724 Haïtiens, 3724 Argentins. 4. Dont 14343 Haïtiens, 8212 Chiliens, 6069 Brésiliens, 4180 Colombiens, 3600 Argentins.

Car dans les années vingt, la Ville-Liberté, Paris-femme ou Paris-nuit, n'est plus l'unique point de référence en matière artistique. C'est plus à Berlin ou à Moscou que s'engagent les révolutions artistiques même si Paris demeure un aimant culturel. Le surréalisme n'est pas à proprement parler un phénomène français, cependant, il est largement un phénomène développé à Paris et perçu comme parisien : à tel point que les dénonciations latino-américaines englobant futurisme, cubisme, dadaïsme et surréalisme définissent ces "ismes" comme " camelote parisienne" 40 . A Buenos Aires, dans les années 19201930, “ el grupo de París " ou “ la escuela de París " ; au Mexique, au début de 1940 l'Exposition Internationale du Surréalisme, puis, plus tard, Octavio Paz, à Paris de 1946 à 1951 ; au Brésil, entre 1925 et 1927, les " manifestations modernistes " et, surtout 
après 1930, avec Vicente do Rego Monteiro, Tarsila de Amaral, et les poètes Jorge de Lima et Murilo Mendes ${ }^{41}$.

Paris conserve durablement sa représentation de centre cosmopolite par excellence. Car les représentations affectant la France évoluent plus lentement que certains des phénomènes envisagés précédemment. Beaucoup de Latino-Américains et plus encore de Français eux-mêmes ne perçoivent pas le processus de récession. Claude Lévi-Strauss écrit: " Comme la plupart de mes contemporains, je n'évaluais pas en 1935 l'ampleur du cataclysme [...] que, vingt et un ans auparavant, l'Europe avait eu la folie de déclencher avec la Première Guerre mondiale, et qui allait la condamner au déclin. Sa puissance semblait toujours intacte, sa domination sur le reste du monde assurée " 42 . Les distinctions les plus élevées couronnent encore la culture française : en 1921, Anatole France reçoit le prix Nobel. Certains signes peuvent même laisser croire au "brillant développement" des relations culturelles universitaires transatlantiques ${ }^{43}$ : au Brésil seulement, les Instituts francobrésiliens de haute culture de Rio de Janeiro (1922) et de São Paulo (1925), la nomination à la faculté de philosophie, sciences et lettres de l'université de l'Etat de São Paulo, en 1934, de six Français sur treize professeurs recrutés en Europe (quatre Italiens et trois Allemands)... Mais, dans ce dernier cas, la prédominance française n'est claire qu'en matière de sciences de l'homme et de la société, manifestant la restriction du domaine d'influence française à la seule aire de culture "non scientifique ". Là où, au début du siècle, la médecine et les sciences exactes françaises rayonnaient, les Etats-Unis dominent désormais ${ }^{44}$.

\section{Des mécanismes liés à la perception de la France}

Les mécanismes d'érosion du modèle français sont liés à quatre problèmes: la supposée unicité du modèle français; l'impact de l'ignorance de l'Amérique latine par la France et de 
la progressive remise en cause en Amérique latine de la supériorité des cultures européennes; ensuite, la distinction accrue entre culture et politique ; enfin, la diffusion de l'idée de décadence de l'Occident ou de la France.

Dans les premières décennies du vingtième siècle, une part des élites latino-américaines reçoit sa formation au sein d'un moule éducatif souvent francophile. Il y a des institutions performantes auc côtés de quelques phares connus : de rares et souvent très jeunes établissements publics d'enseignement primaire ou secondaire français rassemblent en 1933, 2000 élèves dans toute l'Amérique du Sud à Bogotá, Montevideo, Rio, São Paulo ${ }^{45}$ ); d'exceptionnelles institutions très visibles comme à Buenos Aires, depuis 1922, l'Institut de l'université de Paris ; quelques jeunes et brillants représentants de la culture française qui enseignent dans les universités locales, comme à Rio ou São Paulo: Braudel, Lévi-Strauss, Monbeig etc.; et un maillage réseau d'Alliances françaises inégalement serré, très dense en Argentine avec 8 à 9000 élèves en 193946, au Chili, en contraction au Brésil, en expansion modeste au Mexique, mais leur impact est, n'apportant qu'un complément d'enseignement et non un enseignement complet. Car l'essentiel de l'enseignement primaire et secondaire "français " (et supérieur à Lima où longtemps le seul établissement d'enseignement supérieur est contrôlé par des Français ${ }^{47}$ ) est assuré en Amérique latine par des membres des congrégations françaises, y compris dans le Mexique laïc des années trente ${ }^{48}$. Ce sont les plus anciens lieux d'éducation "à la française". Les congrégations françaises sont installées bien avant la séparation de l'Eglise et de l'Etat en France et, après, subventionnées souvent sur les fonds secrets de la République laïque au nom de l'aide au rayonnement culturel national. La législation laïcisant l'Etat mise en place en France entre 1901 et 1905, et en particulier l'interdiction d'enseigner de 1904, a renforcé le mouvement d'essaimage des congrégations françaises. Elles drainent un nombre d'élève très supérieur à l'enseignement directement lié au gouvernement français d'alors. Au Mexique, en 1912, l'enseignement catholique domine au sein des 14 
établissements français. A Cuba en 1940, les congrégations, avec au premier rang les Frères des Ecoles chrétiennes, sont pratiquement seules à diffuser encore un enseignement avec des cours, des programmes et des méthodes d'examens liés à la France. En Argentine, le cas est à peu près identique: au moment de la guerre de 1914-1918, sur les dix établissements "d'enseignement français" de la capitale, huit sont sous la responsabilité de congrégations. En province, la proportion est la même. Ainsi, dans l'ensemble de l'Amérique du Sud, les établissements des congrégations regroupent, au début des années 1930, quelque 150000 élèves ${ }^{49}$. Il s'agit certes d'une petite minorité. Mais, comparé aux effectifs des établissements patronnés par la République ou à ceux " de la société cultivée ", le nombre prend un autre relief. Cet enseignement catholique constitue toutefois pour le modèle dominant républicain français un vecteur ambigu. Il critique volontiers la Révolution française et cette République laïque, "immorale " : en attestent souvent les bibliothèques des écoles congréganistes d'origine française qui rassemblent peu ou pas de livres d'histoire sur la Révolution française, beaucoup d'auteurs proches de l'Action française et d'ouvrages publiés par les éditeurs catholiques parisiens au contenu peu suspect d'affinité républicaine. Ainsi, véhiculée notamment par ces congrégations françaises, demeure en effet l'idée d'une France fille aînée de l'Eglise ${ }^{50}$, plus prompte à reconnaître des valeurs héritées de l'Ancien Régime que celles héritées de la Révolution. L'ambiguïté des effets de l'enseignement dispensé par les congrégations est, dans certains cas, grande

Dans certains pays ${ }^{51}$, les congrégations ont contribué, sur le plan des représentations, à la survivance de l'idée des " deux France " et, sur celui des idées politiques, à la formation d'une droite utilisant idées et valeurs maurrassiennes. La Troisième République a été aussi diabolisée au sein des milieux catholiques (sans que le rayonnement culturel de la France n'en soit nécessairement ou gravement affecté). Ce type de perception fut celle de l'homme d'Etat libéral brésilien Joaquim Nabuco dont les convictions monarchistes se renforcèrent face à 
ce qu'il nommait le "ferment de haine" de la Troisième république $^{52}$. Les religieuses de l'Assomption déclarèrent à l'arrivée de Claudel au Brésil : "Notre bien aimée patrie a besoin de se relever, surtout dans l'esprit des gens de bien " ${ }^{53}$. C'est encore ce que constate un représentant français en Argentine vers le milieu du siècle: "Pendant trop longtemps, la France a été ici considérée comme le type d'une nation libertaire et anticléricale ".

Intellectuels et diplomates français restent en grand nombre imbus d'une axiomatique supériorité culturelle campée derrière trois remparts: volontarisme, ethnocentrisme et ignorance. L'autosuggestion et le pur phantasme de supériorité peuvent être compris comme des réactions au surclassement par le Royaume-Uni et à la défaite face à l'Allemagne en 1870. Le mythe qui fonctionne aussi " comme une sorte de propédeutique au patriotisme par le moyen d'un jacobinisme que n'ébranle aucune incertitude "54. Certes la France demeurait à l'orée du vingtième siècle le centre incontesté de l'innovation culturelle, du cinéma à l'art nouveau (d'origine anglaise, sa diffusion en Amérique latine est parfois assurée via l'Italie et la Belgique). Mais après la Première Guerre mondiale, tel n'est plus le cas. Pourtant le sentiment de supériorité européen demeure très souvent avoué relativement à l'Amérique latine. Le ministre des Affaires étrangères brésiliennes déclarait en 1905: "On se conduit avec le Brésil comme avec une peuplade de sauvages". Finalement, l'Amérique latine paraît devoir sa culture à l'Europe, ce qu'un bon connaisseur de l'Amérique du Sud n'hésite pas à transcrire: " Ce fut une des particularités de l'Amérique latine de solliciter à toutes les époques de son histoire le concours de l'intellectualité européenne - c'est-à-dire française ". ${ }^{55}$. En 1929, le très raciste Abel Bonnard, écrivain et journaliste en donne une limite extrême: " On est touché de voir avec quel empressement les Brésiliens accueillent les Français qui leur paraissent capables de répondre à l'avidité de leurs intelligences. Ils nous montrent les trésors de leur sol et nous demandent ceux de notre culture. Cela rappelle la franchise et la naïveté des anciens 
échanges : ils nous offrent des papillons et nous demandent des idées ${ }^{56}$.

Derrière le phantasme de supériorité, il y d'abord l'ignorance de "l'objet latino-américain": au mieux les connaissances construisent un objet latino-américain global, flou et inexact. "Le Brésil... un détail ", s'insurgeait en 1912 le très francophile Gilberto Amado ${ }^{57}$. Dès le début du siècle, le Groupement des universités et des grandes écoles souhaitait réagir, "tant était grande la méconnaissance de l'Amérique latine par les Français" ${ }^{\prime 58}$. Georges Clemenceau visitant l'Amérique latine avant la Première Guerre parle de " la grâce de ses ignorances " qui lui permet d'avoir une vision sans préjugé ${ }^{59}$. $\mathrm{Au}$ lendemain de la guerre, Ernest Martinenche, secrétaire général du Groupement des Universités, s'interroge: “ [...] N'avons-nous pas fait preuve trop souvent d'une ignorance fâcheuse de ce qui la concernait [l'Amérique latine]? Nos candidats au baccalauréat ne trouvaient pas sans peine [avant la guerre] les noms des capitales des Républiques de l'Amérique du Sud, et les mêmes questions ne laissaient pas d'embarrasser les personnes cultivées ". ${ }^{60}$ Dix années plus tard, dans les années 1930, Claude Lévi-Strauss note qu'avant son départ "le Brésil et l'Amérique du Sud ne signifiaient pas grand-chose " pour lui : "Le Brésil s'esquissait dans mon imagination comme des gerbes de palmiers contournés, dissimulant des architectures bizarres, le tout baigné dans une odeur de cassolette ". Et le directeur de l'Ecole normale supérieure, qui lui proposa son poste d'enseignant à São Paulo, envisageait ce lieu peuplé d'indiens, au moins dans ses faubourgs ${ }^{61}$.

$\mathrm{Si}$ l'on examine la presse française, deux réflexions s'imposent: la présence globale de l'Amérique latine est très faible; seuls les grands pays existent (Mexique, Brésil, Argentine) et, ponctuellement, les pays andins ou d'autres pays en conflit grave surgissent du fonds de l'exotisme. Dans les années 1940, l'acteur et metteur en scène Louis Jouvet, après quatre années passées à sillonner des pays d'Amérique latine qu'auparavant il n'aurait "pas pu situer sur une carte" 62 , distingue mal côtes atlantiques et pacifiques du continent. Cette 
méconnaissance a une traduction linguistique: en 1939 en France, l'espagnol n'est enseigné qu'à $10 \%$ des lycéens français contre $60 \%$ pour l'anglais. La perception dominante de cette Amérique, réductrice et infériorisante, est une perception double. Il y a d'une part l'Amérique des noirs et des indiens dont il n'y a pas grand chose à attendre pour l'avenir de la civilisation ; un diplomate français au Paraguay écrit sans détour dans les années 1930 que "l'Amérique du Sud est, à raison de $80 \%$, peuplée d'Indiens (et de Noirs) c'est-à-dire de semiprimitifs "63. Il y a d'autre part l'Amérique prolongement de l'Europe, parfois perçue comme une "transsubstantiation " de la " civilisation occidentale" ${ }^{64}$. Les termes sont clairs : par le soin des "essaims de la vieille Europe", "un rameau de culture européenne s'est réimplanté dans un sol étranger à son sol natal " 65, écrit ainsi Lucien Febvre. Ce prolongement peut toutefois être conçu beaucoup moins positivement: l'Amérique latine est alors le lieu d'une acculturation, d'un dévoiement à la hiérarchie transparente. C'est aux pays de ce que des publications de l'époque nomment "l'Amérique blanche" que la crédibilité est accordée: " La divergence de climat a augmenté la différence des races. Tandis que l'Argentine, dont l'âpreté au gain se traduit par le labeur, poursuit avec une énergie tenace le développement de son pays et cimente son unité nationale dans un réel sentiment de patriotisme, le Brésil dont l'activité est ralentie par l'influence des tropiques réalise selon la loi du moindre effort des profits immédiats, ne cherche pas à discerner l'intérêt général de la patrie, ignore l'effort et le sacrifice et répand son nationalisme en xénophobie verbeuse "66.

Plus négativement encore, l'Amérique latine est souvent définie comme un ensemble indistinct de "pays neufs, ayant donné à la culture politique universelle le mot de pronunciamientos "67. Et il faut ajouter à cela d'autres stéréotypes "exotiques", du rastaquouère (un mot hispanoaméricain) au ploutocrate exotique. " Je suis brésilien, j'ai de l'or / Et j'arrive de Rio-Janeire [sic]/ Vingt fois plus riche que naguère/ Paris, je te reviens encor! "68, chantait en 1866, dans $L a$ Vie parisienne d'Offenbach, un riche voyageur brésilien à l'allure 
plutôt mi-mexicaine, mi-argentine: une sorte de gaucho à sombrero couvert d'or "volé " et accompagné de "deux petits nègres "- venu à Paris à l'occasion de l'Exposition universelle. Un stéréotype qui ne survit pas à la première moitié du $\mathrm{XX}^{\mathrm{e}}$ siècle: "Faut-il rappeler, écrit-on en France avec nostalgie en 1947, qu'avant cette guerre chaque année nous apportait son tribut de riches Sud-Américains, bronzés de visage et vêtus de clair, qui passaient l'été sur nos plages et l'hiver à Paris, répandant sur nos hôtels et nos établissements de luxe la pluie dorée de leurs pesos, milreis, sucres, soles et autres bolivars "69. La même œuvre lyrique du siècle précédent faisait aussi intervenir un supposé général Malaga (dont le nom relève d'un exotisme plus "spiritueux" et hispanique que latino-américain) de Porto-Rico (" Porto Riche ", comme le mot est fréquemment et négativement utilisé entre les deux guerres en France). Bien loin de disparaître, ce stéréotype issu du siècle précédent s'est au vingtième siècle beaucoup affirmé et diffusé. A une autre extrémité de la culture occidentale, bien que produit belge et diffusée avant 1940 en album à quelque 6000 exemplaires seulement, la bande dessinée d'Hergé, L'oreille cassée, publiée en 1935, témoigne nettement de cette évolution et de la pesanteur culturaliste : l'ouvrage, qui conduit le reporter Tintin au San Theodoros, pays voisin du Nuevo Rico, est un concentré de ces mythologies européennes sur l'Amérique ${ }^{70}$ : sur la toile de fond de la guerre du Chaco (le "Gran Chapo "), rien n'y manque, ni les conspirateurs d'opérette, ni les généraux interchangeables.

Ce sentiment, très fort, du rang supposé supérieur de la culture française ne touche pas seulement les "petits" pays d'Amérique du Sud ou d'Amérique centrale, ou seulement le domaine culturel. En 1926, l'ambassadeur au Brésil déconseille vivement toute démarche de la France pour obtenir des avantages commerciaux. Il estime que c'est au Brésil de prendre l'initiative: "La France n'a pas à assurer le rôle de demandeur "71. Pendant ce temps, Washington et Madrid obtiennent un statut douanier préférentiel et Londres le négocie. L'un des dénominateurs communs des perceptions françaises 
pourrait donc être le suivant: l'Amérique latine est une terre presque sans histoire. Le sous-continent est une terre quasi sans culture, du moins autonome, ou composée essentiellement d'éléments-clichés tel, pour le Brésil, le Carnaval ${ }^{72}$. Même chez un homme de double culture comme Jules Supervielle, l'espace américain n'a ni langage, ni histoire: “Je fais corps avec la pampa qui ne connaît pas la mythologie, [...] Je m'enfonce dans la plaine qui n'a pas d'histoire [...] Et n'a pour toute végétation que quelques talas, ceibos, pitas qui ne connaissent pas le grec et le latin "73. Même chez un Français, bon connaisseur et amateur du sud du continent, comme Georges Bernanos, installé au Paraguay puis au Brésil à partir de 1938, ce sentiment affleure en des termes qui, bien plus amicaux, n'en rappellent pas moins ceux de Gobineau, ministre de France à Rio en 1869 ("Sauf l'Empereur, personne dans ce désert peuplé de coquins "): " Du pays qui m'entoure, écrit-il, je n'attends pas grand-chose, cela va sans dire; il serait vain d'espérer intéresser au sort de la cathédrale de Chartres huit millions de kilomètres carrés d'une forêt qui n'a jamais servi à rien depuis le commencement du monde $" 74$.

La contestation latino-américaine de la primauté de l'Europe plonge au contraire ses racines dans une connaissance "très étendue "75, au sein des milieux cultivés, de la culture du Vieux monde. Certes, la fascination pour l'Europe ne trépasse pas avec la Première Guerre mondiale: "La richesse de la France, c'est d'avoir pu nous donner ses poètes ; la nôtre, d'avoir su les recevoir. Baudelaire, Hugo, Rimbaud, Péguy, Valéry, Claudel, Giraudoux sont pour quelque chose dans le battement de nos cœurs "76, écrit l'argentine Victoria Ocampo. Mais la contestation existait avant la guerre (en 1907): "Un poète américain, aller en Europe? Et pourquoi faire? Un jour viendra où un tel voyage perdra le prestige sacramentel qui fascine aujourd'hui. C'est l'un des symptômes de notre indépendance à demi "77. Et jusqu'au milieu du XXe siècle au moins, elle se fait virulente. Après la Seconde Guerre mondiale, l'argentin Alberto Caturelli, note, dans la lignée de Keyserling: “ L'Europe est le continent de l'être ouvert et total fécondé par l'esprit.[...] 
L'Amérique, au contraire, [...] est le continent de l'être brut, encore non fécondé par l'esprit "78. C'est un peu plus manichéen que le Brésilien Dante Costa, décrivant Paris en 1940 comme "un haut spectacle de perfectionnement ", un lieu où " coule le fleuve de l'intelligence $" 79$.

La critique est parfois nuancée. Ainsi, le Brésilien José Veríssimo dénonce-t-il le statut des "peuples comparses" en matière culturelle; mais il ne condamne pas complètement l'Europe, ne se reconnaît pas dans "l'ibéro-américanisme préconisé par les intellectuels hispano-américains " et imagine que "l'Europe maintiendra encore pendant de longs siècles, peut-être toujours sa suprématie spirituelle" ${ }^{80}$. La critique est cependant souvent plus incisive.

Après la Première Guerre mondiale, le sentiment que l'Amérique latine atteint sa "majorité" affaiblit, désagrège lentement l'idée du péché originel, de la capitis diminutio d'être américain. Auparavant, pouvait dominer l'idée, exprimée au Mexique, selon laquelle, "arrivée tard au banquet de la civilisation européenne, l'Amérique vivait en sautant les étapes, pressant le pas et courant d'une forme à une autre, sans qu'il y eût le temps que mûrisse tout à fait la forme précédente" ${ }^{81}$. Avec l'affirmation de ce sentiment d'une majorité, se développe l'idée que le rythme européen n'est peut-être pas le seul tempo historique possible. Souvent, dans les termes de la critique latino-américaine, la France perd sa spécificité en participant d'un amalgame "européen", d'un rejet globalisant. C'est ce qu'on peut ainsi lire dans la presse colombienne: "Le dédain manifeste de l'Européen pour l'Américain réside dans le faux complexe d'infériorité que nous adoptons en présence de l'homme de l'Occident qui, jusqu'à aujourd'hui, a joui de privilèges d'exception sous le ciel généreux des Amériques ; des privilèges qui en Europe ne sont compensés que par l'ignorance de nos valeurs"82. Comme on peut s'en douter, la diplomatie française analyse tout autrement un phénomène qui met parfois en cause la colonisation européenne: "En présence des divisions idéologiques et des appels à la violence qui troublent le panorama européen, nous voyons se cristalliser, sous le signe 
du panaméricanisme, un nouveau "complexe de supériorité" de l'Amérique latine" 83 .

La déception inspirée par le fonctionnement européocentré de la SDN illustre et renforce les distances prises par l'Amérique latine à l'égard de l'Europe. Une Amérique latine qui représente à la fondation de la Société près de $40 \%$ des membres et qui, de ce fait, espère que sa voix sera entendue. Le Brésil donne l'un des exemples les plus saillants de cette désillusion. Entré en guerre aux côtés des Alliés pendant la Première Guerre mondiale, il souhaita, comme l'Argentine, jouer un rôle significatif au sein de la Société des nations (SDN). Avec ces deux pays, l'Amérique latine, jusque-là éloignée du "Grand monde" diplomatique, selon les termes d'un délégué sudaméricain $^{84}$, espérait entrer en scène, au moins dans les instances de décision de Genève. La presse brésilienne soutint l'action gouvernementale visant à apparaître distinctement aux Palais de la paix. Le Brésil ne put cependant jamais obtenir de siège permanent au Conseil et, représentant isolé du souscontinent après le retrait de la délégation argentine en 1922, il annonce son retrait en 1926.

La perception culturelle est de moins en moins dissociable de la perception politique. Alfonso Reyes, mexicain très francophile, pose cette question prémonitoire en 1937, devant une assemblée de l'Institut international de coopération intellectuelle (IICI) dépendant de la Société des nations, une tribune européocentrée ${ }^{85}$ : "L'Europe est-elle encore en état " de "dicter avec autant de force ses directives spirituelles ?" ${ }^{86} \mathrm{Un}$ autre, d'origine dominicaine, transmet plus sèchement l'impression "de l'homme de la rue" que "l'Europe ne [...] donne plus le bon exemple; ses conseils politiques ne méritent plus d'être suivis " ${ }^{87}$. Plus direct, le quotidien Tiempo, porteparole officieux du gouvernement colombien, remarquait dès 1936: "Le sentiment anti-européen qui a pris dans ce pays un développement si marqué au cours de ces dernières années a été encore accru par l'affaire d'Ethiopie "88. Et, quelques jours plus tard: "Il ne sera pas de trop que [...] s'établisse 
orgueilleusement la belligérance intellectuelle, politique et économique des peuples de notre Continent subjugués jusqu'à aujourd'hui par les formules de la culture occidentale... Il faut voir par exemple comment un Français ignore insolemment l'existence de peuples qui, passionnés de la démocratie, voient dans la Troisième République l'héritière légitime de la Révolution et suivent avec ferveur les orientations de la pensée française. Pourquoi continuerions-nous à ouvrir magnanimement nos portes aux "citoyens du monde" quand nous jouons en Europe le triste rôle du "Métèque" qu'on tolère seulement parce qu'il est la proie convoitée..." ${ }^{89}$ ? $\mathrm{Au}$ Brésil, "l'anthropophagie "90 ou apologie plaisante de l'ogre indigène, inventée à la fin des années 1920, participe d'un phénomène partiellement comparable, sauf que la dévoration des apports étrangers n'est pas là xénophobe mais intégratrice.

L'hostilité politique est rarement irréductible quand l'atmosphère ambiante est faite de sympathie: c'est là l'immense, l'incomparable avantage dont la France a longtemps bénéficié en Amérique latine, affirme en 1934 le très connu professeur de l'Ecole libre des Sciences Politiques, André Siegfried. En d'autres termes, il constate un "débrayage" de plus en plus net entre la représentation de la culture française et la représentation de la politique française; débrayage entre sympathie culturelle et approbation politique. C'est pourquoi, poursuit-il, " on commettrait une erreur si l'on croyait que la sympathie pour notre culture entraîne nécessairement l'approbation de notre politique: il est presque fréquent de rencontrer des hommes qui sont français d'esprit et qui cependant ne se classent pas politiquement parmi les amis de la France "91. Le fonctionnement politique français est un écheveau partout difficile à démêler et à comprendre entre les deux Guerres en Amérique latine. Le parlementarisme français est dans de nombreux pays perçu presque systématiquement à travers le prisme peu flatteur d'agences étrangères, nordaméricaines. L'agence française Havas, au début du siècle en situation très favorable sur le sous-continent, perd entre les deux guerres cette situation. 
En 1941, le président brésilien Vargas, porte-parole d'un courant décelable dans la plupart des pays du sous-continent, " critique vigoureusement l'inefficacité du parlementarisme dans les pays neufs" 92: autrement dit, il considère que le parlementarisme des pays du "vieux continent " n'est pas un modèle à suivre pour l'Amérique latine, et en particulier pour ce Brésil de l'Estado Novo bien éloigné d'un quelconque héritage démocratique. Un universitaire et diplomate péruvien, utilisant des termes qui ne sont pas étrangers à ce qui se dit en France au même moment, explique la chute de la France et la disparition en 1940 de sa démocratie parlementaire par "la décomposition d'un régime dont la faiblesse était en étroite corrélation avec ses énormes vices "93. Cette position est vraisemblablement fortement connotée de convictions religieuses ; mais elle n'est pas isolée ${ }^{94}$.

L'évolution politique en France déçoit. Pour certains groupes la perception n'est, on l'imagine, pas favorable aux choix politiques faits en France en 1936. Ainsi au Brésil ${ }^{95}$. A l'inverse, les élites "de gauche" et certains gouvernements latino-américains populistes ou tentés par une politique d'ouverture à gauche, voire "de gauche", au Chili ou au Mexique par exemple, sont un temps séduits par le gouvernement français de Front populaire. En Colombie aussi. Aux yeux de ces gouvernements, la politique tentée à Paris, en 1936, paraît plus en adéquation avec l'image traditionnelle qu'ils ont de la France ${ }^{96}$. Mais, alors que les opinions latino-américaines sont profondément divisées, élites et gouvernements latinoaméricains, qui avaient à nouveau les yeux fixés sur Paris, sont vivement déçus, avant même Munich, par la politique neutraliste du Front populaire dans une guerre civile espagnole qui enflamme en Amérique latine des divisions politiques existantes ${ }^{97}$.

L'Europe favorise en fait cette évolution dès la fin du dixneuvième siècle. Face au progressisme positiviste, entre 1880 et 1914 apparaissent les premiers syndromes des crises des universalismes: l'Europe est partiellement à l'origine de la 
perception de son déclin. En Espagne, avec la défaite à Cuba et le traité de Paris qui entérine la perte des dernières colonies en Amérique et dans le Pacifique, toute une " génération "98 entame une méditation sur le déclin de son pays.

La France suggère des représentations "correspondantes", non sans lien initial, chez certains auteurs, avec "la crise allemande de la pensée française" 99 . Comme Déroulède, Bourget ou Barrès, Ernest Renan assène : " La France se meurt, [...] ne troublez pas son agonie" ${ }^{100}$ ? Répétées en Europe et parfois directement jusqu'en Amérique latine, la critique du rationalisme progressiste, comme leur antécédente "méditation sur la décadence ", font lentement leur chemin - y compris transatlantique - au vingtième siècle. Albert Demangeon, en 1920, Oswald Spengler, en 1926, développent l'idée plus large du "Déclin de l'Occident". On se souvient du mot fameux de Paul Valéry: "Nous autres, civilisations, nous savons maintenant que nous sommes mortelles". On se souvient moins du livre de ces "critiques impitoyables de la démocratie parlementaire et capitaliste "101 que furent Robert Aron et Arnaud Dandieu, auteurs la même année du Cancer américain, et surtout, pour notre propos, de La décadence de la Nation française (1931). Pourtant ces livres s'inscrivent dans un processus de longue durée où la position française a ses spécificités : en Europe, à l'exception de l'Autriche, c'est bien en France que, dans certains cercles cultivés, la foi dans le progrès s'est le plus effacée, dès la fin du dix-neuvième siècle, devant la prescience du déclin.

Il importe de constater que la méditation sur le déclin s'accompagne souvent d'une perception de l'évolution du monde : le mouvement de la civilisation accompagnerait le trajet du soleil, d'Est (la Grèce, Rome) en Ouest (l'Amérique) ; l'image, affirmée au XIX siècle, avec Tocqueville en particulier, grandit au XX. Bien d'autres Français manifestent ce discours sur la décadence, comme ceux partis chercher, outre-Atlantique ou en Afrique, l'influence régénératrice des peuples et arts dits "primitifs". Antonin Artaud conclut ainsi sa première 
conférence à México, en 1936, par ces mots: "La culture rationaliste de l'Europe a fait faillite et je suis venu sur la terre du Mexique chercher les bases d'une culture magique qui peut encore jaillir du sol "102. Très loin de ces préoccupations, le prestigieux président international des Pen Clubs, Jules Romains, connu, traduit, lu en Amérique latine, détaille en novembre 1938, dans le sillage des Accords de Munich, les " maux" de la France, " d'abord, l'instabilité gouvernementale ", puis " les mauvaises finances ", "la désunion entre les Français, et pis encore peut-être : la manie de l'indiscipline "103 et d'autres "symptômes " encore. Et il ajoute: "Vous allez me dire: "Mais ce que nous faisons est donc si grave, si coupable?" Oui. Vous laissez la France être malade, depuis des années. Vous laissez croire au dehors que la France est malade, et qu'elle le restera ; [...] qu'elle ne tient pas à guérir. Ce qui est peut-être encore plus dangereux. Car être malade, [...] cela peut se prendre en habitude et se supporter... Oui, tant qu'il n'y a pas les autres. Mais laisser croire qu'on est malade, le laisser croire dans ce terrible monde d'aujourd'hui... Alors, cela ne traîne pas" 104. Raymond Aron confirme, dans un autre registre: "J'ai vécu les années 30 dans le désespoir de la décadence française [...]. Au fond, la France n'existait plus. Elle n'existait que par les haines des Français les uns pour les autres "105.

En Amérique latine, le sentiment du déclin français atteint peu à peu une frange non négligeable des élites locales et des classes moyennes en cours de formation. Cela par le biais de ces livres ou d'autres, lus en langue originale (la langue française demeure l'apanage d'une partie de la haute société latinoaméricaine cultivée) ou traduits ; par l'intermédiaire d'articles de la presse latino-américaine ou de revues françaises sur ces livres ; enfin, par le contact direct avec certains intellectuels français véhiculant les mêmes courants d'idées. L'empreinte latino-américaine de cette projection du pessimisme français est nette. L'intégralisme brésilien veut-il "affirmer la valeur du Brésil" ? Il dénonce alors une bourgeoisie "fascinée par la civilisation qui périclite en Europe et aux Etats-Unis" ${ }^{106}$. En 1936 
, l'Argentin Juan B. Terán affirme que la diffusion de cette idée, d'une crise de l'Europe, est en fait le signe d'une fidélité de l'Amérique latine à la pensée européenne: "Quand, en Amérique latine, on parle de déception vis-à-vis de l'Europe, on donne une preuve de fidélité à la culture européenne; ne sont-ce pas les Européens les premiers à avoir parlé de crise européenne ? Vous savez bien à quel point on en est arrivé en Allemagne avec Spengler. En France, il suffit de citer Henri Barbusse et Romain Rolland " 107.

Après celles de Renan ou de Taine, les idées de Maurras sont diffusées ou reçues inégalement en Amérique latine : plus au sud qu'au nord semble-t-il. En fait, il paraît peu raisonnable d'envisager l'influence de la pensée maurrassienne sans prendre en considération que ces idées parviennent aussi, notamment en Argentine, à travers le filtre espagnol, celui de Ramiro de Maeztu et de l'Acción española en particulier, et qu'il est dès lors difficile de distinguer entre parenté idéologique et lien direct. Appliquées ou non à la politique latino-américaine, ces idées antirépublicaines du fondateur du quotidien L'Action française contribuent d'abord à renforcer "un certain préjugé antifrançais " chez les élites les plus conservatrices et catholiques (même après la condamnation de 1926) comme au sein de certains mouvements au discours populiste. Surtout, les bases universalistes de l'image traditionnelle de la France sont ébranlées, y compris auprès d'un public assez large dépassant nettement le cercle restreint des élites intellectuelles. En 1931, paraissait la traduction brésilienne du livre de Léon de Poncins, Les forces secrètes de la révolution: avec La France juive de Drumond, l'ouvrage nourrit l'antisémitisme de Barroso, l'un des doctrinaires les plus antisémites de l'intégralisme brésilien. En 1933, on écrit au Brésil : " La liberté politique a été une conquête de la bourgeoisie pour la bourgeoisie, malgré les promesses universelles de la Révolution française "108.

A partir des années 1920-1930, tandis que l'on peut constater que les écrits français contre-révolutionnaires (Joseph de Maistre, Louis de Bonald...) sont connus en Amérique latine, directement ou par auteur contemporain interposé ${ }^{109}$, l'œuvre de 
Maurras, s'il ne faut pas en exagérer l'influence en Amérique latine, paraît pouvoir constituer une nouvelle matrice pour la gestation (ou la renaissance) éventuelle d'un autre modèle de la France.

Des facteurs externes aux relations France-Amérique latine: les concurrences

On peut esquisser une typologie sommaire des principales concurrences au XXe siècle du modèle dominant français, entre le premier vrai modèle universaliste concurrent que sont le modèle léniniste et la IIIe Internationale, le modèle autoritaire et efficace incarné par l'Allemagne impériale et les fascismes; enfin, celui dont l'expansion n'est pas atteinte, bien au contraire, par la Seconde Guerre mondiale, le modèle continental et linguistique, diffusé par les Etats-Unis et avec le panaméricanisme comme structure de cohésion.

La Révolution russe de 1917 donne corps à un modèle explicitement universaliste et concurrentiel du modèle français. Le modèle communiste, qui ne dédaigne pas d'inscrire la Révolution française dans sa mythologie des origines, possède un écho progressif au sein de certaines élites latino-américaines. Un écho, parfois modeste, largement " créolisé ", selon le mot de l'historien vénézuélien Caballero110, souvent complexe. Le politologue André Siegfried note, en 1934, qu'à terme "la passion spartiate et jalouse de la Russie des soviets " pourrait " détourner peut-être plus encore [que la séduction nordaméricaine] les masses populaires du vieil idéal français "111. La diffusion du communisme sur le continent est rapide mais limitée. Dès 1918, est créé le premier parti communiste latinoaméricain (le Partido Socialista Internacional), en Argentine. Le Brésil suit en 1922, puis le Chili : c'est là, longtemps, le seul pays où le parti communiste constitue un parti de masse. Même dans des contextes politiques moins favorables, comme dans le Venezuela de l'autoritaire Gómez, les idées se diffusent 
rapidement112. En 1919, le Komintern crée sa première section en Amérique latine, à México113. Et, bien qu'inégalement développés selon les pays, il y a 12 partis communistes en 1928, 19 en 1935. Quoi qu'il en soit, ce modèle léniniste se diffuse sans directement beaucoup affecter l'influence du modèle français, bien plus culturel que révolutionnaire.

L'influence allemande est ancienne. L'organisation militaire prussienne et la construction d'une fédération impériale consacrée par la défaite française de Sedan, jointes au développement économique (tardif mais accéléré) et scientifique de l'Allemagne, ont attiré les regards latino-américains avant 1918. Dès les années 1890, certains Français ont fait le constat en Amérique du Sud de "la décroissance" de "l'autorité morale " de la France, jugée notamment par rapport au repère allemand: "On nous tenait toujours pour le peuple le plus vif et, si l'on veut, le plus divertissant de l'univers. Mais s'agissait-il de pédagogie? On invoquait l'Allemagne. De science pure? Encore l'Allemagne. De philosophie? Toujours l'Allemagne. Je visitai [à Santiago du Chili] l'Institut pédagogique, dont le directeur était allemand, et dont la bibliothèque était encore plus allemande que le directeur [...]. Le vieux recteur de l'Université [...] était féru de systèmes allemands. Quand un jeune homme, futur médecin ou futur professeur, désirait achever son éducation en Europe, il allait à Berlin. S'il voulait uniquement s'amuser, il prenait le chemin de Paris "114. Depuis la guerre du Paraguay (1865-1870), des militaires brésiliens séjournent dans l'armée allemande et, comme on l'a signalé, des groupes de stagiaires sont admis dans les Ecoles de guerre allemandes ${ }^{115}$. Dans le domaine économique, l'Allemagne se place, " particulièrement en Amérique latine ", dans le "rôle extraordinairement profitable de courtier international " ${ }^{116}$. Après la guerre, la République de Weimar maintient une active propagande antifrançaise en Amérique latine. Les cercles français bien informés ne sont pas aveugles. En 1922, Jean Giraudoux écrit, dans un roman qu'affectionnait l'historien Jean-Baptiste Duroselle, Siegfried et le Limousin : "Allemagne, ton heure vient peu à peu. Déjà la Bolivie a repris sa mission vétérinaire allemande, déjà [...] 
l'Argentine acclame nos botanistes"117. La présence économique allemande, plus ou moins appuyée sur des "colonies" nombreuses et accrues durant le premier quart du siècle, se renforce rapidement. Les Français, souvent plus soucieux, même sur le champ latino-américain, de l'antagonisme franco-allemand que d'une évaluation globale, en prennent ombrage. Mais c'est, presque toujours, pour réaffirmer haut et fort que le modèle français est intact ${ }^{118}$. L'Allemagne fait pourtant un important effort pour améliorer la connaissance de l'Amérique latine de ses ressortissants: cinq Instituts spécialisés sur l'Amérique latine sont rapidement mis en place et généreusement financés, avec le souci de former tous ceux qui ont quelque chose à y faire.

L'Italie, plus jeune que les nations continentales de l'Amérique latine, monarchie, de faible rayonnement international, ne diffuse pas de "modèle" avant l'instauration du fascisme. Mais les échanges jusqu'à la Première Guerre mondiale sont denses, à commencer par les transferts de population dans le sens Europe-Amérique latine, Amérique du Sud principalement. L'empreinte des cultures d'origine est profonde. Après leur mise en place, les gouvernements totalitaires de l'Italie et de l'Allemagne, surtout (bien qu'il ne faille pas négliger l'exemple précoce du Portugal salazariste pour le fonctionnement de l'Estado Novo au Brésil), exercent une réelle séduction sur une partie de la population latinoaméricaine; en particulier sur les catégories les plus conservatrices des élites et sur les classes moyennes naissantes.

Avant qu'il ne s'effrite tardivement, avec l'évolution du cours de la guerre, le nouveau régime politique italien attire par son goût de l'ordre certains milieux conservateurs qui en approuvent certains résultats apparents pouvant être transposés en objectifs locaux : paix sociale restaurée, accords avec l'Eglise, bolchevisme mis à mal. Plusieurs institutions brésiliennes de l'Estado Novo se sont inspirées de statuts d'homologues fascistes, statuts souvent transmis d'ailleurs à toutes fins utiles par les diplomates italiens : ainsi le Departamento de Imprensa e Propaganda (DIP). La séduction des modèles fasciste puis nazi 
est confortée par une nette croissance des échanges commerciaux. Même si les immigrés allemands sont partout profondément divisés ${ }^{119}$, elle est partiellement relayée par d'actives "colonies": en 1941 plus de 500000 Allemands en Amérique du Sud dont 89000 au Brésil (avec 87 sections du NSDAP en $1937^{120}$ ) et 60000 en Argentine, auxquels il faut ajouter localement 200000 Argentins d'origine germanique avec 120 écoles et plus de deux cents clubs ${ }^{121}$.

Proportion des membres du NSDAP par rapport aux Allemands (citoyens) d'Amérique latine en 1937

\begin{tabular}{|l|c|c|c|}
\hline & $\begin{array}{c}\text { Nombre } \\
\text { d'Allemands }\end{array}$ & $\begin{array}{c}\text { Membres } \\
\text { du NSDAP }\end{array}$ & on \\
\hline Curaçao & 100 & 37 & $1 / 3$ \\
\hline Panamá & 200 & 63 & $1 / 3$ \\
\hline Bolivie & 675 & 169 & $1 / 4$ \\
\hline Chili & 5300 & 985 & $1 / 5$ \\
\hline Colombie & 1400 & 274 & $1 / 5$ \\
\hline Venezuela & 1200 & 221 & $1 / 5$ \\
\hline Cuba & 250 & 42 & $1 / 6$ \\
\hline Honduras & 300 & 41 & $1 / 7$ \\
\hline Equateur & 500 & 58 & $1 / 9$ \\
\hline Guatemala & 2500 & 260 & $1 / 10$ \\
\hline Pérou & 2000 & 201 & $1 / 10$ \\
\hline Paraguay & 2600 & 240 & $1 / 11$ \\
\hline Costa Rica & 645 & 56 & $1 / 12$ \\
\hline Uruguay & 2000 & 143 & $1 / 14$ \\
\hline Mexique & 5500 & 310 & $1 / 18$ \\
\hline Brésil & 75000 & 2903 & $1 / 26$ \\
\hline Argentine & 42600 & 1500 & $1 / 28$ \\
\hline
\end{tabular}

D'après Hans-Adolf Jacobsen, Nationalsozialistische 
Außenpolitik, 1933-1938, Frankfurt am Main, Metzner, 1968, pp. 662 et 665 ; et Anne Saint Sauveur-Henn, ouvr. cité, p. 568.

La séduction des modèles autoritaires est aussi insufflée par une diplomatie et des missions (allemandes) très dynamiques, par la plupart des Latino-Américains formés en Allemagne. La puissance d'attraction regroupe en fait deux caractéristiques différentes. D'une part la séduction politique du modèle autoritaire, en raison de son aspect martial et efficace que de certaines similitudes dans le mode de fonctionnement du pouvoir: outre anti-communisme (et parfois antisémitisme), élévation réciproques des représentations au rang d'ambassades, la collaboration entre les polices politiques ${ }^{122}$ dont le cas le plus évident est, au Brésil, l'expulsion de la compagne germano-brésilienne du dirigeant communiste brésilien Carlos Prestes ${ }^{123}$ ou celle de l'épouse d'un ancien député du Reichstag. D'autre part, la séduction est aussi celle d'une nation humiliée mais forte, capable, par ses propres moyens rassemblés autour d'un chef charismatique, de soulever le joug imposé par des métropoles démocratiques et impérialistes coalisées : un modèle - la référence est exprimée ou non, avouée ou non - pour nombre de Latino-Américains rêvant d'une indépendance nationale mieux assurée. Les régimes antidémocratiques mis en place en Europe par Salazar, Mussolini, Hitler puis Franco, constituent un ensemble de modèles pour nombre de mouvements politiques développés en Amérique latine, dans les années 1930, en Argentine, comme au Chili, ou au Brésil.

C'est à ce stade qu'il faut, d'autre part, bien considérer le phénomène de "créolisation" de ces importations. Les liens à l'Europe sont conscients, inégaux et rarement explicites. Le chef intégraliste, Plínio Salgado, mêle son admiration pour le fascisme italien à des idées venues de l'intégralisme portugais ${ }^{124}$; mais, par nationalisme anticosmopolite, il minimise l'influence fasciste sur l'intégralisme brésilien. Certains gouvernements autoritaires conservateurs d'alors (et les élites qui les accompagnent) puisent dans ces constructions 
autoritaires européennes des idées de symboles, des références, des méthodes ou, peu souvent, des institutions : notamment Ubico au Guatemala (1931-1944), Batista à Cuba, (1940-1952), Uriburu en Argentine ${ }^{125}$ (1930-1932) et Benavides au Pérou (1933$1939)^{126}$.

Dans ces conditions, l'activité diplomatique, les missions militaires et la propagande anti-françaises de l'Allemagne renforcent le déclin du rayonnement du modèle français, un rayonnement incluant la francophonie et la francophilie, expression d'une perception du modèle en tant que tel.

Avant de conclure, il convient de constater que le soulèvement franquiste en Espagne de 1936 est source d'une nette réactivation d'un modèle ancien ${ }^{127}$. Son rayonnement paraît toutefois limité à des élites latino-américaines très traditionnelles, rarement soumises à l'attraction française. Il est donc difficile de considérer le modèle nationaliste espagnol, le modèle de la croisade franquiste, comme un concurrent direct du modèle français. Tout au plus ajoute-t-il, sur un terrain spécifique, sa voix aux autres modèles totalitaires, accentuant leur pesée globale - avant la proposition d'élimination radicale proposée par Vichy.

La Première Guerre mondiale érode en Amérique latine la crédibilité extérieure de l'ensemble des nations européennes. Cette désaffection amorcée de plusieurs modèles européens et, au premier plan, du modèle français est liée aussi à l'affirmation factuelle de la doctrine panaméricaine de Monroe, puis de sa variante ultérieure plus fine qu'est la "politique de bon voisinage". Même si, de France, la prise de conscience est lente $^{128}$. Et même si la connaissance commune de l'Amérique latine parait peu progresser aux Etats-Unis entre les deux guerres. Mais, par exemple, la pénétration des agences de presse nord-américaine (Associated Press, United Press) dans les circuits latino-américains d'information est constante entre les deux guerres et se trouve en situation hégémonique partout pendant la Seconde ${ }^{129}$. Dans un tout autre domaine, celui de la formation, dès 1918 le constat peut être fait que les étudiants 
latino-américains "fréquentent aussi bien Harvard que l'université de Paris "130 et que les fondations multiplient leurs propositions en direction de l'Amérique latine. De plus, l'accroissement de la diffusion du livre classique en espagnol et en portugais est nette aux Etats-Unis ${ }^{131}$ et, en 1926, il y a 175 institutions du supérieur donnant des cours aux Etats-Unis sur l'Amérique latine. Des Instituts culturels sont créés: en 1925, premier du genre, l'Institut culturel argentino-nord-américain; puis l'Institut Brésil-Etats-Unis, l'Institut péruano-nordaméricain, l'Institut Benjamin Franklin à México montrent la voie suivie ailleurs. Signe d'une prise en compte nouvelle, le Secrétariat d'Etat crée en 1938 une division des relations culturelles, avec des objectifs, précisément formulés et dans cet ordre : enseignement, livre, radio, servir d'intermédiaire pour les organismes privés. Le continent, jugé "indécis" dans son positionnement idéologique, paraît la priorité ${ }^{132}$. En 1942 le Département d'Etat possède autant d'attachés culturels que d'ambassades sur le continent. L'administration de l'Education met sur pied une Division des relations éducatives interaméricaines. Enfin, au début de la guerre, la Présidence se dote le 16 août 1940, en dépendance directe, d'un Bureau de la coordination interaméricaine remarquablement efficace : l'Office for Coordination of Commercial and Cultural Relations between the American Republics en 1940, Office of the Coordinator of Inter-American Affairs l'année suivante. Les " bandits mexicains" d'Hollywood disparaissent sur sa pression des écrans ${ }^{133}$ tandis que Donald fait, avec Walt Disney en personne, une entrée remarquée en Amérique latine et que de nombreux intellectuels latino-américains entreprennent le déplacement vers le nord. Que peuvent, pendant la guerre, les brochures de la France libre face au contrôle nord-américain de la fourniture en papier et face à Selecciones, l'édition espagnole du Reader's Digest qui " se vend à la criée dans toute l'Amérique latine ${ }^{134}$ ou face aux 500000 exemplaires mensuellement diffusés en Amérique latine de la revue de propagande En Guardia ( $E m$ Guarda au Brésil) -et ce n'est pas, loin de là, l'unique publication $^{135}$ ? Il faut ajouter à tout cela le rôle des organismes 
de l'Union panaméricaine et ce qui est fait dans ce cadre et qui s'accélère beaucoup sous la présidence de Franklin D. Roosevelt, dans le cadre de la "politique de bon voisinage". Avec les termes de l'époque, non dénués du sentiment viscéral de supériorité de la culture intellectuelle française, la remarquable ouverture "géographique" de la culture d'André Siegfried lui permet, dès 1934, d'exprimer deux "menaces" pouvant atteindre "l'influence de la France sur " l'Amérique latine ${ }^{136}$ : " La première serait celle d'une démocratisation de l'Amérique du Sud, conformément à l'idéal matériel et quasi mécanique du vingtième siècle. Quand les peuples d'aujourd'hui se démocratisent $[\ldots]$, ils élèvent sans doute leur niveau de vie [...], mais la tonalité moyenne par l'éviction des élites sociales tend uniformément à devenir plus vulgaire. Or pareille évolution ou révolution ne les rapproche nullement d'une France traditionnelle qui, quoique profondément attachée à l'égalité, a conservé, jusque dans ses couches populaires, je ne sais quel raffinement d'esprit et de goûts. [...] L'élite lisait nos livres, se faisait donner nos pièces de théâtre, buvait nos vins fins; la masse, parvenue, fréquentera les cinémas américains, lira des romans policiers traduits de l'anglais, boira de la bière ou des alcools brutaux. [...] La France, à cet égard, est un peu démodée, et on nous le dit assez. L'établissement de la démocratie dans le monde ne se fait plus sous notre inspiration. C'est l'Amérique du Nord qui, depuis une vingtaine d'années, a pris la tête, dès qu'il s'agit de la démocratie du confort, et son matérialisme ne travaille pas pour nous [...]. "La seconde menace, qui réside dans la mécanisation et la commercialisation de la vie, est à peine moindre; elle est même plus directe, plus immédiate. Quand les pays, même latins, se mettent à l'école des méthodes américaines, il est naturel qu'ils se tournent vers les EtatsUnis "137. Et " c'est moins par goût que par nécessité ", conclut André Siegfried.

Enfin, dans le seul domaine linguistique, partout ailleurs, la langue française paraît demeurer, dans beaucoup de grands pays, la langue étrangère la plus étudiée en Amérique du Sud 
entre les deux Guerres : dans les années vingt nettement, mais partout de manière plus discutée dans les années trente.

$\mathrm{Au}$ début du siècle, la supériorité de la langue française était un dogme intangible en France: "la langue française importe à l'ordre général de la civilisation "138. Au Brésil, Georges Clemenceau observait avant la Première Guerre mondiale qu'il pouvait "se livrer en toute confiance au plaisir de parler comme un Français à des Français, sans que rien lui vînt avertir des particularités d'une âme étrangère à laquelle il fût tenu de s'accommoder"139. Lorsqu'en 1923, en qualité de Président de la commission des Affaires étrangères de la chambre des députés, Maurice Barrès visite l'Argentine, c'est le même langage qui est tenu ${ }^{140}$. Le français continue d'être un élément discriminant de la culture des élites. Dans les années trente, Fernand Braudel et Claude Lévi-Strauss enseignent encore en français à l'université de São Paulo. André Siegfried constate à l'issue de conférences au Brésil en 1937 : “ Je sais par expérience qu'on peut parler français, devant des auditoires de 2 ou 300 personnes et être compris exactement comme on le serait en France [...]. On peut même parler dans le détail, de notre vie politique ou littéraire, et toutes les précisions semblent intéresser le public ${ }^{141}$. Et, encore, lorsque la troupe de l'Athénée-Louis Jouvet représente du théâtre français en français dans la plupart des pays du continent entre 1941 et 1944, la compréhension de la langue ne paraît pas un problème trop sérieux pour les assemblées -choisies il est vrai.

Mais ces constats clairs et renouvelables à l'infini ne doivent pas masquer le déclin de la langue française avant la Seconde Guerre mondiale en Amérique latine. D'une part il n'est plus que dans peu de pays au premier rang des langues étrangères parlées par les membres jeunes des élites, à la fois parce que les congrégations enseignantes n'ont pu renouveler leur vivier d'enseignants en France et du fait des restrictions des programmes scolaires latino-américains ${ }^{142}$. D'autre part, la situation semble plus délicate au nord qu'au sud du souscontinent latin, avec des inégalités considérables d'un pays à l'autre. Tandis qu'en Argentine le français demeurerait "la" 
langue de la culture encore durant la Seconde Guerre mondiale, les exemples de Cuba, pays sous une tutelle nord-américaine plus ou moins directe depuis 1898, et du Mexique sont probants : la langue française connaît un net recul dans le nord de l'Amérique latine, en particulier dans cette zone, dite de sécurité des Etats-Unis, que dessinent les interventions militaires nord-américaines, jusqu'au canal de Panamá et aux Guyanes. Elle cède du terrain partout dans le monde, officiellement (dans les programmes scolaires ou universitaires) et pratiquement. La langue diplomatique par excellence n'étaitelle pas au dix-neuvième siècle le français? Encore en 1943, lorsque Roosevelt et Vargas se rencontrent à Natal, la langue utilisée lors des échanges par les Présidents est le français, seul dénominateur commun entre eux. Néanmoins, dans la première moitié du vingtième siècle, le monopole est, peu à peu, défait. Car, face au français, "l'enseignement de l'anglais s'est grandement développé " depuis le début du siècle. Une partie, variable selon les pays, des élites latino-américaines manifeste des traits d'anglophilie au-delà de la stricte étiquette: qu'on songe, par exemple, à la multiplication de clubs sportifs pour gentlemen locaux à la peau claire. Mais le processus qui conduit la langue anglaise à élargir son domaine d'influence s'accélère surtout après la Première Guerre mondiale -en provenance cette fois des Etats-Unis plus que des îles britanniques. Dans les années trente, le français et l'anglais "sont sur le même pied " 143 dans les programmes scolaires de beaucoup de pays ; dans certains, les deux langues sont obligatoires, dans d'autres le choix est possible. Et pendant la Seconde Guerre mondiale, les choses évoluent plus rapidement encore. Significativement néanmoins, ce qui, à la fin de la guerre, est évoqué par la diplomatie française n'est déjà plus la nécessité de préserver dans le sous-continent la primauté du français; l'objectif désormais visé consiste à "obtenir que le français soit mis sur le même pied que l'anglais dans les programmes officiels". Pis, la diplomatie française commence à percevoir la difficulté d'un tel objectif et la nécessité d'attendre que le pays " ne se trouve plus 
sous la domination économique exclusive des Etats-Unis": "Toute tentative prématurée n'aurait d'autre résultat que d'inquiéter les Américains, et de les pousser à obtenir quelques avantages supplémentaires d'ordre culturel ou autre, pour consolider leurs positions $" 144$.

Outre ce qui est déjà énoncé, la diffusion de l'anglais tient au moins à six raisons : la formation à l'étranger qui est de plus en plus dirigée vers les Etats-Unis ("tels jeunes gens de l'aristocratie sud-américaine $[\ldots]$ qui naguère eussent fait leur éducation à Paris, vont maintenant chercher à New York, à Boston, en Californie, la culture financière ou technique des temps nouveaux "145); la formation dans le pays (l'anglais est exigé dans un nombre croissant d'examens entre les deux guerres); les modes intellectuelles (depuis le début du siècle s'est affirmé un "engouement pour toutes les habitudes, théories, doctrines" nord-américaines, particulièrement pour les questions économiques); les nécessités de la vie courante (recrutement local des entreprises anglaises et américaines). Les deux autres raisons expliquent que l'anglais se diffuse au-delà des élites économiques, politiques et sociales: accessibilité du livre anglo-saxon; diffusion du cinéma américain. Le public latino-américain, qui éprouve des difficultés croissantes à se procurer des ouvrages en français, "veut lire les livres déversés en abondance sur le marché par les éditeurs nordaméricains "146 : il n'en existe par exemple dans tout le Chili aucune librairie française et le principal libraire vendant le livre français à Santiago, un Portugais, ferme ses portes en 1916 tandis qu'il existe là quatre librairies anglaises et deux allemandes ; le paradoxe est alors qu'on ne trouve plus à Quito, Bogotá ou Caracas de livres français en vente mais qu'on en parle dans la presse ! Un intellectuel et ancien ministre mexicain José Vasconcelos constate: "Pour se convaincre du fait qu'aujourd'hui il y a moins de personnes qui lisent la langue de Corneille qu'il y a vingt ans, il suffit de parcourir les bouquinistes [de México]. Les livres les moins chers sont ceux écrits en français " ${ }^{147}$ (Paul Rivet ouvre en réaction à la fin de la guerre une librairie française). Logiquement, "les bibliothèques 
privées [...], même les mieux fournies ", accusent avant la Seconde Guerre mondiale "un certain retard " par rapport à la production contemporaine ${ }^{148}$. La dernière raison contribuant à expliquer la diffusion de la langue anglaise en Amérique latine, auprès d'un public incluant une classe moyenne émergente est le cinéma: si, au début des années vingt, le cinéma français pouvait figurer en bonne place dans les salles de spectacle latino-américaines (et peut-être surtout sud-américaines), dans les années trente, la situation s'est radicalement modifiée: le public veut désormais comprendre, avec l'anglais, rien moins que " $95 \%$ des films projetés" ${ }^{149}$. Ce chiffre, donné par le service des Euvres françaises à l'étranger, ne noircit pas la réalité si l'on compte par projection et non pas par film. En 1936, 76 \% des films programmés en Argentine et $91 \%$ au Brésil sont d'origine anglo-saxonne. Dans ce dernier pays, $87 \%$ des films projetés sont alors nord-américains; même au Mexique, cette part dépasse les trois quarts de la programmation de la capitale $(73,2 \% \text { en 1940 })^{150}$. Quoi qu'il en soit, en 1936 et 1937, pour 20 films étrangers projetés en Argentine et au Brésil, 1 peut être français quand 15, dans le meilleur des cas, sont nordaméricains ${ }^{151}$. Au Mexique, la situation est à peine moins fragile : sur 20 films projetés à México, 14,5 nord-américains, 1,5 français, avec cependant plus d'un film français pour neuf nordaméricains (films français : 8,2 \% des films programmés, 9,0\% des films étrangers). De plus, les films nord-américains sont, la presse en témoigne, programmés plus ou moins simultanément partout en Amérique, tandis que les films français parviennent tardivement en Amérique latine et souvent sur des copies très usagées, moins chères.

\section{La faible mise en œuvre de la propagande française}

Face à cette expansion d'autres références européennes ou nord-américaines, les gouvernements de la Troisième République n'ont compris que tard et mal, par rapport à 
l'Allemagne en particulier, l'intérêt de l'organisation des colonies nationales à l'étranger, de la diffusion culturelle, de la propagande ou des nouveaux moyens de communication. Et l'Amérique latine apparaît très loin des priorités.

Pendant la Première Guerre mondiale, la France a bien mis en œuvre du bout des lèvres une "propagande" via une "Maison de la Presse " dont l'axe essentiel était anti-allemand. Mais, au lendemain de la guerre, les gouvernements s'empressèrent de restreindre les activités de la propagande officielle française, parce que la culture française n'avait, pensait-on alors, nullement besoin de cette béquille et par dédain pour cet art de la publicité. Entre les deux Guerres, la gestion des budgets publicitaires allemands, en particulier ceux destinés à la presse écrite, des commerçants ou industriels locaux, est centralisée sous la responsabilité d'un fonctionnaire allemand. La diplomatie française se dote, moins tardivement que modestement, de structures culturelles. Elle s'organise certes dans un mouvement qui est commun à la plupart des puissances européennes, mais elle suit plus qu'elle n'entraîne et, au total, demeure peu étoffée avant 1939. En Amérique latine, à la veille de la Seconde Guerre mondiale, la radio française est l'une des radios étrangères les moins audibles; dans nombre de régions, c'est celle que l'on capte avec le plus de difficultés. La radio est un média américain. En 1920, la première station de radio commerciale voyait le jour aux Etats-Unis; en 1921, commençaient les premières émissions de radio au Mexique.

Les Etats-Unis exercent d'emblée une influence déterminante, en fournissant aussi bien les modèles d'émission que la très grande majorité du matériel d'émission ou de réception ( $99 \%$ des postes vendus au Mexique en $1933^{152}$ ). Aussi bien pour les " heures étrangères " des radios latino-américaines que pour les émissions en ondes courtes, la France n'est pas un concurrent très crédible des autres nations étrangères. Pendant la seconde Guerre mondiale, la situation évolue très fortement en faveur des Etats-Unis. A partir de 1941, le Bureau de Coordination des Affaires interaméricaines réussit à mettre discrètement en lien, pour la fourniture de journaux 
d'information traduits, près de 200 stations de radio latinoaméricaines avec des radios des Etats-Unis. Même pour le Brésil, où la langue a freiné ces échanges, en 1943, 92 stations de radio utilisent des émissions nord-américaines ${ }^{153}$. Il ne faut cependant pas exagérer l'impact -considérable- de ce nouveau moyen de communication: l'information proprement dite ne dépasse pas 10 à $15 \%$ des programmes ${ }^{154}$; surtout, le nombre d'appareils est relativement restreint dans certains pays à la fin de la période étudiée ; en conséquence, l'auditoire (très urbain mais par contre non restreint aux seules élites) est encore modeste $^{155}$ (1/4 de la population en 1950 au Mexique, seulement $6 \%$ au Guatemala). L'apparition en 1924 de la radio sur ondes courtes ouvre définitivement des horizons internationaux à ce nouveau moyen de communication : des horizons longitudinaux d'abord, entre les Etats-Unis et le reste du continent. Mais pas seulement: en février 1934, les premiers programmes en espagnol de Radio Berlin reçus en Amérique latine montrent que le domaine des ondes hertziennes est devenu un enjeu très important des propagandes. Tard venue, la radio française, elle, est beaucoup moins bien reçue que toutes les émissions en provenance des Etats-Unis. Mais elle aussi partout moins nette que les radios allemandes ou anglaises.

La propagande officielle n'est pas mieux armée. Le service des Euvres des Affaires étrangères est la plus ancienne structure administrative française spécialisée dans le domaine des relations culturelles internationales. En 1910, l'initiative administrative en matière culturelle fait une entrée discrète au ministère des Affaires étrangères : c'est le bureau des Ecoles et des œuvres françaises à l'étranger, lequel fonctionne avec une ou deux personnes seulement. Malgré le dédain pour " l'art de la réclame ", la Première Guerre mondiale renforce l'idée d'une nécessaire action concertée: consacrant la spécialisation des activités du ministère, un Office de relations publiques et de propagande est d'abord créé en 1915. La structure que l'on appelle bientôt pudiquement la "Maison de la presse " est née. Après la guerre le gouvernement commence, au contraire, par désorganiser la Maison de la presse devenue, sous le ministère 
Clemenceau, Commissariat général de l'information et de la propagande. Même les francophiles latino-américains se sentent délaissés: " [Nous] demandons d'urgence à tous les échos -pour nous et pour la France qui a retrouvé [avec la guerre] ses filles perdues- une politique française en Amérique latine" ${ }^{156}$. En 1920, le Bureau des écoles devient le service des Euvres françaises à l'étranger. Il est chargé de toutes les questions concernant "l'expansion intellectuelle de la France au dehors "157 (le British Council n'est créé qu'en 1934). Ses moyens sont limités et la part américaine a longtemps diminué (de $11 \%$ au lendemain de la guerre, $10 \%$ en $1920,6 \%$ en 1924 à $6,6 \%$ en 1930), ne connaissant une récupération relative que dans les années 1930 (8,7 \% en 1933). Encore s'agit-il pour une bonne part des Etats-Unis, c'est-à-dire, de l'ancien allié que l'on a souhaité cultiver activement pendant la guerre et au-delà. Et si l'on examine le détail de l'activité française de diffusion culturelle, le constat de ce retard, relativement à d'autres régions du monde, est manifeste. Le service des Euvres réalise néanmoins à la veille de la guerre des efforts visibles pour développer les liens culturels entre Amérique latine et France : on constate ainsi le début de fonctionnement "d'interchanges universitaires", au Mexique notamment à partir de 1938 - mais au rythme très faible de deux boursiers mexicains par an.

Répartition géographique du budget du service des Euvres des Affaires étrangères, 1921-1933

\begin{tabular}{|l|l|c|c|c|}
\hline & Europe & Orient & Extrême-Orient & Amérique \\
\hline 921 & $43 \%$ & $29 \%$ & $17 \%$ & $11 \%$ \\
\hline 924 & $28,5 \%$ & $\begin{array}{c}54,5 \% \text { (dont 34,5\% } \\
\text { yrie-Liban) }\end{array}$ & $11 \%$ & $6 \%$ \\
\hline 933 & $38 \%$ & $\begin{array}{c}57 \% \text { (dont 27 } \% \\
\text { Syrie-Liban) }\end{array}$ & $6,3 \%$ & $8,7 \%$ \\
\hline
\end{tabular}

Source : Les Affaires étrangères et le corps diplomatique français, ouvr. cité, p. 451. 
Enfin, deuxième structure administrative de soutien à l'action culturelle à l'étranger, l'Association française d'action artistique (AFAA) est créée en 1922, sous ce nom en 1934. Organisme d'exécution du ministères des Affaires étrangères et de celui de l'Education nationale, il est chargé de réaliser des manifestations artistiques internationales. S'il œuvre pour la première fois hors d'Europe, sur le continent américain, en 1925 à New York puis, l'année suivante, au Canada, c'est en 1932 seulement que son domaine atteint l'Amérique latine, avec la subvention d'une tournée de "cours-conférences" de Marguerite Long au Brésil. Puis rien jusqu'en 1938.

L'AFAA et l'Amérique latine, 1922-1940

1932 : tournée Marguerite Long (Brésil)

1938 : exposition de dessins français à Bogotá.

1939 : représentations de la Comédie française à Buenos Aires, Rio, Montevideo

1939 : exposition d'art français "De David à nos jours" à Buenos Aires

1940 : exposition "Cent ans de peinture française" à Montevideo, Rio de Janeiro (avant San Francisco)

1940 : représentations du théâtre du Vieux-Colombier en Amérique du Sud (Buenos Aires, Montevideo...)

1940 : exposition de livres, gravures, dessins, aquarelles à Santiago (Chili).

Sources: AMAE-N, dossiers divers.

L'Amérique latine apparaît bien comme une préoccupation tardive, devenue assez importante seulement dans l'immédiat avant-guerre ${ }^{158}$ : l'Amérique latine est loin du centre des préoccupations françaises.

Il est difficile toutefois de conclure ce tableau de la mise en œuvre de la propagande française sans mentionner le travail 
accompli, de la déclaration de guerre à la défaite, sous les auspices du Commissariat national à l'Information, avec des résultats sensibles: " La propagande étrangère au Brésil - nordaméricaine, française et britannique - s'est beaucoup développée et sur une large échelle, laissant la nôtre à une distance extrêmement grande ", constate un diplomate italien ${ }^{159}$. Si rien en ce cas n'attire alors plus la sympathie que la France menacée, les enjeux sont toutefois ailleurs, ce qui enferme les limites de cette sympathie dans un cadre restrictif. Le même diplomate explique en effet que l'attitude internationale de ce pays n'est en fait dépendante ni d'une opposition interne à l'autoritarisme, ni d'une inféodation culturelle et politique à la pensée française, ni de la propagande ou de l'argent des Alliés, ni de préoccupations liées à l'expansion allemande; seul importe vraiment le lien aux Etats-Unis.

A partir de 1945, économiquement, culturellement, en dépit du "rejeu " de francophilie constaté, l'Amérique latine s'est un peu plus éloignée de l'Europe. Le modèle français a progressivement cessé d'être perçu en tant que modèle universaliste, se rétractant à sa dimension simple de modèle national. Comment ne pas penser à dresser, dans ces conditions, un parallèle avec ce que dit Pierre Nora de l'évolution en France du modèle de nation? "Le modèle classique, providentialiste, universaliste et messianique, s'est progressivement délité, dès les lendemains de la guerre de 1914 à la fin de la guerre d'Algérie, qui [...] a scellé la conscience définitive du déclin ". "Le remaniement profond de la conscience nationale auquel nous assistons aujourd'hui suppose un tout autre modèle de nation. Il correspond à la stabilisation de la France au rang des puissances moyennes et à son insertion dans un ensemble européen, conflictuel, mais pluriel et pacifié ${ }^{160}$.

Tout modèle culturel, même à vocation universaliste, est périssable. Non seulement la durée d'existence et la validité du modèle sont limitées, mais cette limitation même en fait l'intérêt principal: "Le naufrage est toujours le moment le plus significatif", écrivait Fernand Braudel. La Seconde Guerre mondiale a fonctionné apparemment comme une catharsis des 
imaginaires. Vichy, avec un armistice fondateur qui déconcerte les Latino-Américains francophiles, avec un reniement de la république et des idées xénophobes que l'on pardonne à la France en fonction de son occupation partielle, focalise progressivement sur sa représentation tout ce que l'on jugeait en inadéquation avec le modèle traditionnel français. Volontiers jugé prisonnier, Vichy parvient avec difficulté à affirmer en Amérique latine qu'il représente "la vraie France". Vichy se contente en fait une représentation d'accident de conjoncture. A l'inverse, la France libre et ses réseaux constitués en Amérique latine contribuent à réactiver l'image traditionnelle. Des Français non diplomates, souvent bon connaisseurs des réalités nationales américaines, prennent en main, avec la diplomatie de la France libre, la gestion de l'image de la France, bénéficiant en outre de l'antithèse présentée par Vichy et du fait que les démocrates latino-américains soucieux de ne pas trop s'afficher trop près des Etats-Unis trouvent en la résistance française un emblème bien moins dangereux. Ainsi, pour une génération. Se constate alors le "rejeu " en plusieurs temps de la francophilie: une relance pendant la guerre, liée à la guerre, résultant de la remobilisation des énergies autour de la France libre et d'une image "épurée " de la France ; et cette France est de nouveau pensée comme "éternelle", selon une abstraction qui est aussi l'une des clés de voûte de la symbolique gaullienne. Le "rejeu " est renforcé par le rayonnement de la France internationalement restaurée- du général de Gaulle et par le développement de l'activité culturelle institutionnelle française dans les années qui ont suivi la guerre. Mais cette réactivation de la francophilie dure approximativement ce qu'a duré la génération ayant connu la période de la guerre.

L'étude de la crise du modèle français contribue moins à la distinction entre legs initial et construction mémorielle qu'à l'analyse de cette dernière. Au vingtième siècle l'image du legs est elle-même fondatrice du modèle, le legs importe dans une certaine mesure moins que ses représentations, de part et d'autre de l'Atlantique, et que leurs évolutions. Nous ne sommes pas loin ainsi d'une définition de l'aire de puissance, culturelle et 
politique, de la France. En Amérique latine, la France, définie comme pays de culture par excellence dans la seconde moitié du dix-neuvième siècle, est, depuis 1918, peu à peu perçue aussi comme un conservatoire culturel, comme un pays de culture non active. La France ne dément pas nécessairement cette perception, tentée qu'elle est par un "essentialisme culturel ", "tourné vers le passé, privilégiant dogmatiquement la culture classique "161. En outre, même si elle admet sur certains terrains spécifiques que l'Amérique latine puisse être autonome, la France a globalement des difficultés à s'ouvrir à une relation culturelle qui ne soit pas assise sur un axiome double d'antériorité et de supériorité, et sur l'idée connexe de filiation des autres cultures.

Analysée dans un premier vingtième siècle qui s'arrêterait avec l'armistice de 1940, la crise du modèle français apparaît comme le temps de la prise en considération réciproque du chemin descendant sur lequel sont engagés les liens entre France et Amérique latine ; un chemin au cours duquel les deux parties enregistrent, pour la notion de modèle, le resserrement d'une acception politique et culturelle à la seule acception culturelle. A partir de l'été 1940 et de manière plus formalisée à partir de 1945, plus encore dans les années 1960, vient le temps de la prise en charge, du côté français, de l'exportation du modèle. Cette prise en charge est marquée par la création de la Direction générale des relations culturelles (en avril 1945) et par le déploiement d'une diplomatie culturelle sur une base spécifique à la France: "l'autonomie du culturel ". Il s'agit d'endiguer un déclin dont on a mis plus longtemps en France qu'en Amérique latine à percevoir la réalité.

L'Europe sort-elle de la scène latino-américaine tandis que l'Amérique latine tend à entrer sur la scène mondiale ? Des mots comme L'Amérique latine entre en scène, maintes fois utilisés depuis plusieurs décennies, proviennent surtout de deux phénomènes parfois associés : les espoirs de développement du sous-continent latino-américain représenté comme "ExtrêmeOccident", et le pessimisme cultivé par l'Europe. Néanmoins, en termes plus modérés et pour le seul exemple français, le 
vingtième siècle constitue-t-il le couchant d'un modèle universaliste, le modèle français ? Un auteur plusieurs fois cité, André Siegfried, remarquait, au seuil des années soixante, en pensant aux Etats-Unis, " qu'il se pourrait que, dès aujourd'hui, le centre de gravité de l'Occident ne soit plus en Europe " 162 ...

$\mathrm{Au}$ vingtième siècle, l'Amérique latine fonctionne comme un laboratoire dans lequel il est aisé, d'une part, de voir s'effriter le monopole d'une Europe "élaboratrice " de modèles ; d'autre part, de constater, dans certains cas, l'inversion du sens de transfert. Cela ne signifie d'ailleurs pas qu'Europe et Amérique latine ne participent pas encore d'un même ensemble culturel supra-régional: ce dernier peut simplement nécessiter une définition plus complexe. Dans un ordre international peu à peu mondialisé, l'Amérique latine est devenue depuis le milieu de ce siècle un creuset d'élaboration d'éléments de modèles (ré-) exportés vers l'Europe. Pensons à la littérature. Pensons aussi que le modèle révolutionnaire, depuis la prise de pouvoir de Fidel Castro à La Havane, s'est pour une génération renversé : les Européens qui maniaient des années 1960 aux années 1980 l'adjectif révolutionnaire cherchaient des références outreAtlantique. Pensons aussi à cette théologie de la libération, même si elle a partiellement été élaborée dans certains cercles en France (et en Belgique), qui a été perçue comme une mise en œuvre latino-américaine. Pensons enfin, en matière de théories sur le développement, à la théorie de la dépendance forgée par des Latino-Américains et qui a fait le tour de la planète. Ainsi, avec la littérature, les théories sur le développement, l'exemple révolutionnaire cubain, les années 1960 ont manifesté qu'était devenu possible, dans le court ou le moyen terme, le renversement de la direction d'exportation d'éléments constitutifs de modèles entre l'Europe et Amérique latine. 
ROLLAND, Denis. The Exemplary crisis of the European Model in Latin America: the olden roots of the image of the French Political and Cultural Model. História, v.27, n.1, p. 283-344, 2008.

Abstract: The French and Francophiles have been glorified for more than a century now because of the decisiveness of the French influence in the birth of new states throughout the Latin American continent. Thus, today we can see a constant return to this New World: of the revolutions of independence and of the birth of nation-states of the Americas up to the present day. The history of Latin America would have progressively approximated the cultural sphere, shaking itself free of the European influence, in particular of France. In our opinion, this phenomenon requires an analysis, which we intend to accomplish here.

Keywords: Francophiles; French influence; Latin America

\section{NOTAS}

Abréviations utilisées pour les citations de sources:

AMAE : Archives du ministère français des Affaires étrangères, Paris.

AMAE-N : Archives du ministère français des Affaires étrangères, Nantes.

BNF, FJ, TAL: Bibliothèque Nationale de France, Fonds Jouvet, Tournée en Amérique latine.

CPDOC-FGV : CPDOC, Fundação Getúlio Vargas, Rio de Janeiro.

NAW, RG 59 : National Archives, Washington. State Department files.

1. Albert Salon, L'action culturelle de la France dans le monde, Paris, Nathan, 1983.

2. Cf. Romain Gaignard, Les Aveyronnais dans la pampa, fondation, développement et vie de la colonie aveyronnaise de Pigüe, Argentine, 1884-1974, Toulouse, Privat, 1977 ; Patrice Gouy, Pérégrinations des Barcelonnetes au Mexique, Grenoble, PUG, 1980 ; Marie-Jeanne Paoletti, L'émigration corse à Porto Rico au XIXe siècle, thèse de doctorat, Aix-Marseille 1, 1990...

3. Cf. Denis Rolland, La crise du modèle français, Marianne et l'Amérique latine, XXe S., Rennes, Presses universitaires de Rennes, 2000. 
4. Georges Lomné, Frédéric Martinez \& Denis Rolland dir., avec Annick Lempérière, Les modèles de l'Europe en Amérique latine, Paris L'Harmattan, 1998.

5. Ici européenne. Lucien Febvre, "Premier entretien public", in Le Nouveau Monde et l'Europe, Neuchâtel, Ed. de la Bâconnière, 1955, pp. 204-205.

${ }^{6}$. Ou construction mentale globale et collective. Cf. Jacques Le Goff, L'imaginaire médiéval, Paris, Gallimard, 2e édition, 1991, préface p. II.

7. Cf. Michel Vovelle (dir.), L'image de la Révolution française, 3 vol., Pergamon Press, 1989; le $\mathrm{n}^{\circ} 54$ de Caravelle (Toulouse, 1990) et le $\mathrm{n}^{\circ} 10$ des Cahiers des Amériques latines, (Paris, 1990); La Révolution française et l'Amérique latine, Paris, La Découverte, 1989.

${ }^{8}$. Le Brésil impérial a suivi une voie de compromis entre modernité et tradition.

9. Cf. François Chevalier, L'Amérique latine, de l'indépendance à nos jours, Paris, PUF, 2e éd., 1993, p. 157.

10. Francisco García Calderón, cité par Mateo Pérez-Pacheco, "La culture française en Amérique latine ", Bulletin de l'Amérique latine, 06/07-1921, p. 272.

11. Charles Morazé, Les trois âges du Brésil, essai de politique, Paris, Armand Colin, 1954, p. 24.

12. François-Xavier Guerra, "Les avatars de la représentation au XIXe siècle ", in Georges Couffignal, Réinventer la démocratie, Le défi latinoaméricain, Paris, FNSP, 1992, pp. 49-84.

13. François-Xavier Guerra, art. cité, pp. 54 et 62.

14. François-Xavier Guerra, art. cité, p. 70.

${ }^{15}$. Mario Carelli, "Les Brésiliens à Paris de la naissance du romantisme aux avant-gardes ", in André Kaspi et Antoine Marès (dir.), Le Paris des étrangers, Paris, Imprimerie nationale, 1989, p. 287.

${ }^{16}$. Cf. l'introduction de François Chevalier, L'Amérique latine, ouvr. cité, 2 e éd., p. 434 sq.

17. La pensée de Spencer et de Darwin paraît l'emporter sur celle de Comte en Uruguay et en Argentine. Au Brésil, Alberto Sales, idéologue de la république libérale, est un héritier du darwinisme social via Spencer et le modèle de la République idéale est fourni par les EtatsUnis.

18. John Lynch, "La Iglesia católica en América Latina 1830-1930", in Leslie Bethell ed., Historia de América latina, vol. 8, Ed Crítica, Barcelone, 1991, p.91. 
19. Pierre Bouretz, "D'Auguste Comte au positivisme républicain ", in Pascal Ory (dir.), Nouvelle histoire des idées politiques, Paris, Hachette, 1987, p. 301.

20. Guzmán Blanco décrète en 1870 l'éducation primaire publique et obligatoire. L'université est réformée sous l'influence du positivisme.

21. Daniel Pécaut, Entre le peuple et la nation, Les intellectuels et la politique au Brési, Paris, MSH, 1989, pp. 25.

22. La Colombie est un cas particulier: à la fin des années 1880 le positivisme est balayé par le conservatisme politique.

23. Afrancesado: "francisé", avec un fort sens d'acculturation. Cf. Annick Lempérière, Intellectuels, Etat et société au Mexique, Paris, L'Harmattan, 1992, p. 38.

${ }^{24}$. Luis Alberto de Herrera, La Revolución Francesa y Sud América, F. Sempere, Valencia, 1910.

25. E. Bradford Burns, "Introduction", Les Révolutions dans le monde ibérique (1766-1834), II. L'Amérique, Talence, P.U. Bordeaux, 1991, pp. 7 et 13.

26. Cité par Gilbert Freyre, Terres du sucre, Paris, Gallimard, 1956, p. 158.

27. François-Xavier Guerra, " préface " à C. Parra Pérez, Miranda et la Révolution française, Caracas, Banco del Caribe, 1989, p. IV.

28. Cf. Marie-Danièle Demélas, L'invention politique, Bolivie, Equateur, Pérou au XIXe siècle, ERC, Paris, 1992, pp. 73 sq. et 189-190.

${ }^{29}$. Ernest Renan, Qu'est-ce qu'une nation?, in Euvres complètes, Paris, Calmann-Lévy, 1947, p. 891.

30. A México, il n'entretient dans les années trente qu'une activité nominale.

31. Avec d'autres pays dont le Paraguay, le Brésil choisit en 1919 une mission française.

32. Silvio Azuela, cité par Jorge Silva, Viajeros franceses en México, México, 1947, p. 22.

33. D'Argentine au moins, des Allemands font toutefois le voyage pour combattre en Europe.

34. Cf. Patrice Gouy, ouvr. cité.

35. Cf. Denis Rolland, Vichy et la France libre au Mexique, Guerre, cultures et propagandes, Paris, L'Harmattan/Publications de la Sorbonne, 1988, p. 88.

36. Source: AMAE, G.39-45, VAm, Cuba, Politique intérieure (205), Barois, 6-12-1940. 
37. AMAE-N, Rio légation, série $A, n^{\circ} 209$,.

38. Cf. Frédéric Mauro, "French firms and financing of brazilian industrialization", communication multigraphiée XIth Int. Congress of Economic History, Milan, 1994, p. 13 et sa bibliographie.

39. Cf. Roberto Cova, Raúl Gómez Crespo, Arquitectura marplatense, el pintoresquismo, Buenos Aires, Resistencia, 1982.

40. Luciana Stegagno Picchio, "Jorge de Lima et Murilo Mendes : les deux faces du surréalisme au Brésil ", in Nouveaux mondes, autres mondes, Surréalisme et Amériques, Paris, Lachenal \& Ritter, 1995, p. 190.

41. Luciana Stegagno Picchio, art. cité, pp. 193-202.

42 . Claude Lévi-Strauss, Saudades do Brasil, Paris, Plon, 1994, p. 18.

${ }^{43}$. Cf. Hugo Suppo, in D. Rolland (dir.) Le Brésil et le monde, Paris, L'Harmattan, 1998, pp. 127-148.

44. En 1923, par exemple, l'Institut Rockefeller fait un important don à la faculté de médecine de São Paulo.

${ }^{45}$. Les lycées de Buenos Aires et México sont créés en 1934 et 1937.

46. Pour Buenos Aires, Jacques Soppelsa, "Francia en la Argentina : un contexto privilegiado", in Francia en la Argentina, Buenos Aires, Manrique Zago ed., 1995, p. 35.

47. L'université catholique de Lima dirigée par les Pères du Sacré-Cœur.

48. Il existe aussi certains collèges privés laïques (Buenos Aires, Pernambouc...).

49. Gilles Matthieu, Matthieu G., Une ambition sud-américaine, politique culturelle de la France 1918-1939, Paris, L'Harmattan, 1991.

50. Cf. René Rémond, "La fille aînée de l'Eglise ", in Pierre Nora (dir.), Les lieux de mémoire, III. Les France, 3. De l'archive à l'emblème, Paris, Gallimard, 1992, pp. 541-581.

51. Au Brésil, par exemple, comme au Canada.

52. Cité par Mario Carelli, "Les Brésiliens à Paris ", art. cité, p. 288.

53. AMAE-N, Rio légation, série $A$, carton $n^{\circ} 209$, Congrégation des religieuses de l'Assomption à Claudel, 24-02-1917.

54. Pascal Ory, "Le mythe de Paris, Ville-Lumière, dans les années 1900", in Pierre Milza, Raymond Poidevin (éd.), La puissance française à la "Belle Epoque", Mythe ou réalité?, Bruxelles, Complexe, 1992, p. 134.

55. "La librairie française en Amérique latine", in Actes de la Première semaine de l'Amérique latine, Lyon, 1916, pp. 285- 301.

${ }^{56}$. Abel Bonnard, Océan et Brésil, Paris, Flammarion, 1929, p. 74. 
57. Gilberto Amado, Mocidade no Rio e Primeira viagem a Europa, Rio de Janeiro, José Olympio, 1956, p. 221.

${ }^{58}$. Paul Appel en 1908 cité par Charles Lesca, "Histoire d'une revue", in Hommage à Ernest Martinenche, Etudes hispaniques et américaines, Paris, D'Artrey, 1939, p. 430

59. Georges Clemenceau, Notes de voyage dans l'Amérique du Sud, Argentine, Uruguay, Brésil, Paris, Hachette, 1911, p.

60. Ernest Martinenche, "L'Amérique latine et la guerre ", , in Bulletin de l'Amérique latine, Paris, 03/04-1918, p. 164.

61. Claude Lévi-Strauss, Tristes tropiques, Paris, Plon, 1955, pp. 49-50.

62. Louis Jouvet, Prestige et perspective du théâtre français, Paris, Gallimard, 1945, p. 21.

63. AMAE, Am18-40, d. gnx, 43 (191), note de M. Pierrotet (ministre au Paraguay) pour le directeur politique, 13-06-1938.

64. Georges Duhamel s'interroge sur ce point en septembre 1936 à Buenos Aires, Europa-América Latina, Buenos Aires, IICI, 1937, p. 4.

${ }^{65}$. Lucien Febvre, "Les Lumières de Clio", art. cité, p. 14.

66. AMAE-N, Rio de Janeiro, B, 4 (209), Conty, 24-06-1926.

67. Cf. Denis Rolland, Vichy et la France libre au Mexique, ouvr. cité..

${ }^{68}$. Jacques Offenbach, livret de Henri de Meilhac et Ludovic Halévy, La Vie parisienne, 1866.

69. Raymond Chevrier, Pierre Waleffe, L'Amérique du Sud, Paris, Gründ, 1947 , p. 8.

70. Plus nettement que Les 7 boules de cristal (1943), et Le temple du soleil (1944-1946).

71. AMAE-N, Brésil, fds B n³, 333, 13-10-1926, Conty.

72. Le Nord-Estin Jorge Amado l'a bien compris qui publie en 1932 un premier roman intitulé Pays du Carnaval.

${ }^{73}$. Jules Supervielle, Débarcadères, Paris, Gallimard, 1922, p. 13

74. Georges Bernanos, "Journal 1940", Le lendemain c'est vous !, Paris, Plon, 1969, pp. 15-16.

75. Ernest Martinenche, “ L'Amérique latine et la guerre ”, art. cité, p. 164.

76. Cité par Louis Jouvet, ouvr. cité, p. 60.

77. La Obra de Ricardo Rojas, Buenos Aires, La Facultad, 1928, p. 67. Cité par Christiane Séris, "Microcosmes dans la capitale ou l'histoire de la colonie intellectuelle hispano-américaine à Paris entre 1890 et 1914 " in Kaspi A. \& Marès A. (dir.), Le Paris des étrangers, Paris, Imprimerie nationale, 1989, p. 308. 
78. América bifronte, Buenos Aires, Ed. Troquel SA, 1956-1957 ; cité par M.T. Martinez Blanco, Identidad cultural de Hispanoamérica, Madrid, U.C.M., 1988, p. 227.

79. Dante Costa, Itinerario de Paris, Rio de Janeiro, Leitura, 2ed éd., 1945, pp. 19 et 187.

80. José Veríssimo, Cultura, literatura e política na América Latina, (João Alexandre Barbosa éd.), São Paulo, Brasiliense, 1986, p. 185.

${ }^{81}$. Alfonso Reyes, ouvr. cité, p. 7. Transposé au passé.

82. Ruy Blas (Carlos Lleras Camargo) in Tiempo, Bogotá, 16-05-1936.

83. AMAE, Am18-40, Colombie, 5 (211), 23-08-1939, L. Vasse, chargé d'affaires. Texte souligné par nous.

84. Cité par Jorge Guillen, in Bulletin de l'Amérique latine (déc.janv.1921), p. 70.

${ }^{85}$. Cité par Céline Arcade, La politique extérieure du Brésil vue par les diplomates français, mémoire de maîtrise, université de Rennes 2, 1995.

${ }^{86}$. Alfonso Reyes, in Europe-Amérique latine, ouvr. cité, p.1.

87. Pedro Henríquez Ureña, in Europe-Amérique latine, ouvr. cit., p. 123.

88. Tiempo, Bogotá, 31-04-1936.

89. Tiempo, Bogotá, mai 1936, cité par AMAE, Am18-40, Colombie, 5 (32/34), A. Blanche, 17-05-1936.

90. Oswald de Andrarde, Manifeste anthropophage, 1928.

91. André Siegfried, Amérique latine, ouvr. cité, p. 153.

92. AMAE, VAm., Brésil (23), Saint-Quentin, 22-12-1941.

93. F. Barreda Laos, ouvr.cit., p.65.

94. CPDOC/FGV, OA, 400102/3, 910, Cyro de Freitas-Valle à Getúlio Vargas, 25-08-1940.

95. AMAE, Am18-40, Brésil, 108, 01-08-1936, Magnan de Bellevue.

96. Michael R. Marrus, Les exclus, les réfugiés européens au XXe siècle, Paris, Calmann-Lévy, 1986, p. 146

97. Cf. Mark Falcoff, Fredrick B. Pike eds, The Spanish Civil War, 19361939 : American Historical Perspectives, Lincoln, 1982.

98. Cette très littéraire "génération de 98" compte notamment Unamuno, Azorín, Valle Inclán, Baroja, Machado, Maetzu, Benavente...

99. Titre d'un ouvrage fondateur de Claude Digeon, ouvr. cité.

${ }^{100}$. Cité par Paul Déroulède, L'Alsace-Lorraine et la fête nationale, Paris, 1910, p. 7.

101. Raymond Aron, Mémoires, Paris, Julliard, 1983, vol. 1, p. 139.

${ }^{102}$. Antonin Artaud, Surréalisme et révolution, in Fuvres complètes, t. VIII, Paris, Gallimard, 1964, p. 171 sq. 
${ }^{103}$. Jules Romains, "Appel au pays" (24-11-1938 Radio Paris PTT), in Cela dépend de vous, Paris, Flammarion, 1939, pp. 56-57.

104. Jules Romains, déclaration citée, in ouvr. cité, pp. 52-53.

105. Raymond Aron, ouvr. cité, vol. 1, pp. 137 sq et vol.2, pp. 941 sq.

${ }^{106}$. Cité par Helgio Trindade, La tentation fasciste au Brésil dans les années trente, Paris, M.S.H., 1987, p. 127.

107. In Europa-América latina, ouvr. cité, p. 122.

108. Plínio Salgado, Miguel Reale, "A cartilha do Integralismo Brasileiro", in A Doutrina Integralista, Porto Alegre, AIB, s.d., p.13.

${ }^{109}$. L'un des idéologues de l'intégralisme brésilien les cite.

110. Manuel Caballero, Latin America and the Comintern, 1919-1943, Cambridge, Cambridge U.P., 1986.

${ }^{111}$. André Siegfried, Amérique latine, ouvr. cité, p. 156.

${ }^{112}$. Juan Bautista Fuenmayor, La difusión de las ideas socialistas en las carceles gomecistas, Caracas, Universidad Santa Maria, 1982.

113. Cf. Manuel Caballero, ouvr. cité. Pour le Mexique, Barry Carr, "Marxism and Anarchism in the Formation of the Mexican Communist Party, 1910-1919", HAHR, mai 1983.

${ }^{114}$. André Bellesort, art. cité, p. 247.

${ }^{115}$. Cf. Manuel Domingos Neto, art. cité, p. 47.

116. Armand Petitjean, La concurrence internationale et les sympathies françaises en Amérique latine, Paris, Imprimerie nationale, 1918, p. 24.

117. Jean Giraudoux, ouvr. cité, p. 138.

${ }^{118}$. Louis Mandin, L'Intransigeant, cité par la Revue de l'Amérique latine, $\mathrm{n}^{\circ} 1,01-1922$, p. 78 .

119. Anne Saint Sauveur-Henn, Un siècle d'émigration allemande vers l'Argentine, 1853-1945, Böhlau Verlag, Köln, 1995, p. 551 sq.

${ }^{120}$. L.P. Motta, H.J. Barbosa, El nazismo en el Brazil, Buenos Aires, Claridad, 1938, p. 58 et A.-A. Jacobsen, Nationalsozialistische Aussenpolitik, 1933-1938, Frankfurt am Main, Metzner, 1968, p. 550.

121. Cité par Orlando L. Peluffo, in Dictionnaire diplomatique, Paris, Académie diplomatique internationale, 1947, p. 106.

122. R.A.S. Seitenfus, ouvr. cité, pp. 86-90.

${ }^{123}$. Olga Benario (née Kruger) sera fusillée à Ravensbruck en 1942.

${ }^{124}$. Parmi les auteurs portugais qui l'ont influencé, João Ameal, Hipólito Raposo, Rollo-Preto, Oliviera Salazar, Antonio Sardinha.

${ }^{125}$. Parvenu au pouvoir, il rejette l'étiquette "fasciste" et affirme que le fascisme, "doctrine étrangère" est " inappropriée pour l'Argentine. 
126. En 1934, report des élections, politique mêlant répression et paternalisme. En 1936, annulation des élections, dissolution de l'Assemblée et installation d'un gouvernement composé de militaires.

127. Cf. les travaux de Lorenzo Delgado Escalonilla (CSIC, Madrid) dont Imperio de papel, Acción cultural y política exterior durante el primer franquismo, Madrid, CSIC, 1992.

${ }^{128}$. Armand Petitjean, art cité, 1916, p. 152.

${ }^{129}$. Gerson Moura, ouvr. cité, p. 33.

130. Armand Petitjean, ouvr. cité, p. 6.

131. Armand Petitjean, ouvr. cité, p. 13.

132. Inter-American Cultural Relations, Washington, US Government Printing Office, 1939.

133. 122 films en portugais sont patronnés par le Bureau (Moura, ouvr. cité, p. 42).

${ }^{134}$. BNF, FJ, TAL, Cuba (34), J.-C. Abreu à Jouvet, 12-10-1943.

${ }^{135}$. Au sommet de la diffusion de cette revue. Cf. Moura, ouvr. cité, p.35.

${ }^{136}$. En utilisant le mot Amérique du Sud dans le sens d'Amérique latine.

137. Amérique latine, ouvr. cité, pp. 156-157.

138. Discours à l'Alliance française de Paris en 1888, cité par Marc Blancpain in Rapport d'activité de l'Alliance française, Paris, 1970, p. 27.

139. Georges Clemenceau, Notes de voyage dans l'Amérique du Sud, Argentine, Uruguay, Brésil, Paris, Hachette, 1911, p. 63.

${ }^{140}$. Maurice Barrès, cité par Jacques Soppelsa, art. cité, p. 32.

141. FNSP, 1 SI 11 dr.2 sdra, " Impressions du Brésil ", p. 22.

${ }^{142}$. L'Action française, Paris, 17-03-1935.

143. AMAE, Am18-40, d. gnx, 38, (101), s.d. [1934].

144. AMAE, G.39-45, Alger CFLN, Pérou, vol. 1299 (210), 21-08-1944, Dayet.

${ }^{145}$. André Siegfried, Amérique latine, ouvr. cité, p. 157.

146. AMAE, Am18-40, d. gnx, 38, (101), 1934. "Les librairies ont toutes un rayon de livres anglais et américains très bien approvisionné, avec les dernières nouveautés arrivées en avion. Il n'en est pas de même pour le livre français".

${ }^{147 .}$ Novedades, México, 11-02-1944.

${ }^{148}$. Emile Henriot, ouvr. cité, pp. 215-216.

${ }^{149}$. Statistique pour l'Amérique du Sud (AMAE-N, SOFE, n436). 
${ }^{150}$. M. L. Amador \& J. Ayala Blanco, Cartelera cinematográfica (19401949), México, UNAM, 1982, p. 372.

151. Cf. C. de Cicco, Hollywood na Cultura Brasileira, São Paulo, Convívio, 1979.

152. NAW, RG 165, G-2 Report, "Communications - Wire and Radio ", report 5760, 25-10-1934, p. 3.

${ }^{153}$. Gerson Moura, ouvr. cité, p. 45.

154. José Luis Ortiz Garza, La guerra de las ondas, ouvr. cité, p. 21.

${ }^{155}$. Cf. NAW, RG 229, box 346, " Surveys ", W. C. Longan, 29-08-1942.

${ }^{156}$. Mateo Pérez-Pacheco, art. cité, p. 277.

157. Cité par Les Affaires étrangères et le corps diplomatique français, tome II, Paris, CNRS, 1984, p. 393.

${ }^{158}$. Source: AMAE-N, EA, d. 141, notice sur l'AFAA, s. d.

159. Umberto Grazzi, Rio de Janeiro, 03-01-1940, cité par Amado Luiz Cervo, As relações históricas..., ouvr. cité, p. 163.

${ }^{160}$. Pierre Nora, "Comment écrire l'histoire de France", Les lieux de mémoire, III, Les France, 1. Conflits et partages, Paris, 1992, pp. 29-30.

${ }^{161}$. Hichem Djaït, L'Europe et l'Islam, Paris, Seuil, 1978, p. 24.

162. Préface au Rapt de l'Europe de Diez del Corral, Paris, 1960.

Artigo recebido em 03/2008. Aprovado em 07/2008. 